\title{
Quasi steady state and dynamic hygrothermal performance of fibrous Hemp and Stone Wool insulations: Two innovative laboratory based investigations
}

\author{
Eshrar Latif a,b,1, Simon Tucker ${ }^{b}$, Mihaela Anca Ciupala a , Devapriya Chitral \\ Wijeyesekera ${ }^{c}$, Darryl John Newport ${ }^{a}$, Marian Pruteanu ${ }^{d}$ \\ a University of East London, London, UK, ${ }^{\mathrm{b}}$ Centre for Alternative Technology, UK, ${ }^{\mathrm{C}}$ University Tun \\ Hussein Onn Malaysia, Johor, Malaysia, d "Gheorghe Asachi" Technical University of lasi, Romania
}

\begin{abstract}
Moisture and heat management properties of Hemp and Stone Wool insulations were studied by mounting them between a hot and a cold climate chamber. Both insulations were exposed to identical hygrothermal boundary conditions. Quasi steady state and dynamic tests were carried out at a range of relative humidity exposures. The likelihood of interstitial condensation was assessed and equivalent thermal conductivity values of the insulations were determined. The adsorption-desorption isotherms of the insulations were also determined in a dynamic vapour sorption (DVS) instrument. It was observed that the likelihood of condensation was higher in Stone Wool insulation than in Hemp insulation. Hemp insulation performed better in managing moisture due to its high hygric inertia and water absorption capacity. It was observed that the equivalent thermal conductivity of Stone Wool insulation was dependent on enthalpy flow and phase change of moisture. The equivalent thermal conductivity of Hemp insulation was close to its declared thermal conductivity in dynamic conditions when high relative humidity exposures were transient. In quasi steady state boundary conditions, when the insulation was allowed to reach the equilibrium moisture content at ranges of relative humidity, there was a moisture dependent increase of thermal conductivity in Hemp insulation.
\end{abstract}

\section{Introduction}

Standard assessment methods to determine the hygrothermal properties of building materials provide information of their behaviour and performance in steady state conditions. However, hygrothermal boundary conditions are dynamic in reality [1]. Building materials with identical thermal conductivity values may have significantly different heat capacities, materials with similar vapour diffusion resistance factor may have significantly different moisture adsorption capacities and materials with similar

\footnotetext{
${ }^{1}$ Corresponding author. Tel.: +44 7540606063. E-mail address: e.latif@bath.ac.uk (E. Latif).
} 
porosity may have different values of tortuosity. All of these can result in varied hygrothermal behaviour of the materials in dynamic boundary conditions.

Hygrothermal properties of thermal insulations have been studied by a number of researchers. Goto et al. [2] measured the relative humidity and temperature across the depth of a vapour open wall in a climate chamber. The relative humidity of the climate chamber was gradually raised from $20 \%$ to $90 \%$ at $5^{\circ} \mathrm{C}$ while external temperature remained $23^{\circ} \mathrm{C}$. The resulting relative humidity inside the vapour open envelope did not exceed more than $80 \%$. Pavlik and Černý [3] tested the suitability of formulated stone wool insulation in the inner surface of masonry envelope as a vapour open system using a climate chamber. They concluded that it was possible to use internal insulation in a vapour open masonry system by applying a specific water absorbing plaster on the internal surface of the masonry. Arnaud [4] developed a 'cell of exchange' where one side of the experimental sample was facing the temperature and relative humidity of the laboratory and the other side was facing the controlled temperature and relative humidity of the climate box. Arnaud compared the hygrothermal properties of Hemp-lime concrete, aerated autoclaved concrete and vertically perforated bricks and observed dampened fluctuation of temperature inside the Hemp-lime sample. Moisture dependent thermal conductivity of Hemp, jute and flax insulation at different relative humidity exposures was measured by Korjenic et al. [5] and that of glass and mineral wool insulations was measured by Abdou and Budaiwi [6] using heat flow meters. Abdou and Budaiwi observed $216 \%$ increase of moisture dependent thermal conductivity of mineral wool insulation when $15 \%$ weight based moisture was introduced into the insulation. However, measuring moisture dependent conductivity in a hotbox or hotplate in a steady state condition may cause moisture migration and moisture gradient in the sample [7] and wrapping the sample in foil or 
similar covering will not represent the heat flux in a vapour open construction. As much as twenty two times increase of moisture dependent thermal conductivity of hydrophilic mineral wool insulation was observed by Jerman and Černý [8] using transient thermal analyser. Laboratory based experiments were also carried out to compare cellulose and Stone Wool insulations in high internal moisture load and low external temperature $[9,10]$ and the impact was more apparent in thermal properties than in hygric ones.

The in situ work carried out by Latif et al. [11] at ranges of internal relative humidity showed that Wood-Hemp insulation performed significantly better than Stone Wool insulation in reducing the frequency and likelihood of interstitial condensation while the equivalent $U$-value of both insulations remained close to the $U$-value calculated from the manufacturers' declared thermal conductivity values. Nicolajsen [12] assessed thermal transmittance of cellulose loose-fill insulation (with and without a vapour barrier) and Stone Wool insulation (with vapour barrier) in a north facing timber frame wall at $20^{\circ} \mathrm{C}$ internal temperature and $60 \%$ internal relative humidity. For similar thickness, the thermal transmittance value of cellulose was $0.14 \mathrm{~W} / \mathrm{m}^{2} \mathrm{~K}$ and that of Stone Wool was $0.12 \mathrm{~W} / \mathrm{m}^{2} \mathrm{~K}$. Southern [13] tested a masonry wall with internal glass fibre insulation and internal vapour barrier. It was found that during summer time condensation could occur in the inner surface of the vapour barrier. Similar observations were also made by Derome and Saneinejad [14]. Rasmussenand and Nicolajsen [15] studied the performance of insulated roofs .with vapour barrier and walls without vapour barrier in real life conditions for two years. Cellulose, wood-fibre, flax fibre materials and mineral insulations were assessed in terms of moisture management. The moisture conditions in the insulation did not create any risk of mould growth. An insitu study [16] of application of vapour open mineral wool internal insulation on solid brick walls showed that there was no deterioration in the 
hygrothermal performance of mineral wool insulation. Walker and Pavia [17] investigated the in situ thermal performance of thermal paint, aerogel, cork lime, hemp lime, calcium silicate board, timber fibre board and PIR board on a historic brick wall. The insulations were applied to walls facing different orientations and hygric behaviour of the insulations was not assessed. Cork lime and hemp lime decreased the wall Uvalue by $45 \%$ and $36.9 \%$, respectively. In situ performance of thermal insulation materials was also studied by [18-20].

To date no laboratory based experimental work has been reported on assessing the hygrothermal behaviour of Hemp insulation in dynamic hygrothermal boundary conditions in comparison to that of a conventional fibrous insulation material.

This paper reports the results of a number of laboratory tests that are carried out to assess the hygrothermal properties of Hemp and Stone Wool insulations of identical thermal conductivity and differing in vapour permeability by $21.7 \%$, under both dynamic and quasi steady state boundary conditions. The reason for selecting two different hygrothermal protocols is to study if there is any difference in moisture and heat management of insulations when these are exposed to quasi steady state boundary condition incorporating prolonged exposure to each step of relative humidity conditions compared to when they are exposed to fully dynamic boundary conditions involving frequent changes in relative humidity and temperature.

\section{Theory}

\subsection{Moisture adsorption and diffusion}

The moisture storage capacity of insulation materials as a function of relative humidity at a constant temperature can be characterised by its adsorption isotherm [21]. The 
rate of moisture transfer through an insulation at a constant temperature can be characterised by its vapour permeability or vapour diffusion resistance factor [22].

\subsection{Liquid water absorption}

Porous materials, in direct contact with liquid water, absorb water by capillary forces. The ratio of water flux through the free water surface and the square root of time is expressed as the water absorption coefficient.

\subsection{Condensation, vapour pressure, dew point temperature}

Condensation can occur in a surface when the surface temperature is equal or less than the dew point temperature of the vapour in touch with the surface. Moisture concentration inside different insulations can be compared in terms of the corresponding vapour pressures. Dew point temperature and vapour pressure can be determined from temperature and relative humidity values using Equations 1 and 2, respectively:

$$
\begin{aligned}
\mathrm{T}_{\mathrm{D}} & =\left[\left(\frac{v}{100}\right)^{\frac{1}{8}}\right](112+0 . \mathrm{TT})+0.1 \mathrm{~T}-112 \\
\mathrm{e} & =6.11 * 10^{\frac{\left(7.5 * \mathrm{~T}_{\mathrm{D}}\right)}{\left(237.7+\mathrm{T}_{\mathrm{D}}\right)}}
\end{aligned}
$$

Where, $T_{D}=$ dew point temperature $\left({ }^{\circ} \mathrm{C}\right), v=$ relative humidity $(\%), T=$ temperature $\left({ }^{\circ} \mathrm{C}\right), \mathrm{e}=$ actual vapour pressure $(\mathrm{hPa})$

\subsection{Equivalent thermal conductivity}

For the purpose of this paper, 'equivalent thermal conductivity' is defined as the thermal conductivity value determined either in quasi steady state or in dynamic hygrothermal boundary conditions, based on the method of determining in-situ U-value of building elements in dynamic hygrothermal boundary conditions. According to ISO 9869 [23], U-value can be determined from the following equation: 


$$
\mathrm{U}=\frac{\sum_{j=1}^{n} q_{j}}{\sum_{j=1}^{n}\left(T_{i j}-T_{e j}\right)}
$$

Where, $U$ is thermal transmittance $\left(W /\left(m^{2} . K\right)\right), j$ is the number of individual measurements, $q_{j}$ is total density of heat flow $\left(\mathrm{W} / \mathrm{m}^{2}\right), T_{i j}$ is total internal temperature $\left({ }^{\circ} \mathrm{C}\right)$ and $\mathrm{T}_{\mathrm{ej}}$ is total external temperature $\left({ }^{\circ} \mathrm{C}\right)$. Equivalent thermal conductivity can be determined using the following equation:

$$
\lambda \text { equi }=\mathrm{d} \cdot \mathrm{U}=\mathrm{d} / \mathrm{R}
$$

Where, $\lambda$ equi is equivalent thermal conductivity $(\mathrm{W} /(\mathrm{m} . \mathrm{K}))$, $d$ is insulation thickness $(\mathrm{m}), \mathrm{R}$ is thermal resistance $\left(\left(\mathrm{m}^{2} . \mathrm{K}\right) / \mathrm{W}\right)$ of insulation. $\mathrm{R}$ can be calculated from the following equation:

$$
R=R_{T}-R_{1}-R_{s i}-R_{s e}
$$

Where, $R_{T}$ is the total thermal resistance of the component, $R_{s i}$ is the internal surface thermal resistance, $R_{1}$ is the design thermal resistance of acrylic, $R_{s e}$ is the external surface thermal resistance.

ISO 9869 outlines the following likely errors in heat flux measurements: $5 \%$ error due to the calibration of the heat flux and temperature sensors, $5 \%$ error due to the random variation caused by difference in thermal contact between the sensors and the surface when one heat flux sensor is used, $2 \%$ operational error due to the modification of isotherms by the placement of heat flux sensors, $5 \%$ error due to variations in temperature and heat flux over time and when the test wall is not in direct contact with sunlight. Another $5 \%$ error is introduced to the thermal transmittance value or $\mathrm{U}$-value measurement due to the temperature variations within the space and difference between air and radiant temperature. Thus, the total error in U-value measurement can be calculated from the following equation: 
Total error in U-value measurement $=\sqrt{5^{2}+5^{2}+2^{2}+5^{2}+5^{2}}=10.2 \%$

\subsection{Design value of thermal conductivity}

The design value provides an estimate of the thermal conductivity of an insulation material during service conditions and is extensively used in engineering practices [24]. According to ISO 10051 [25], when the effect of moisture on thermal conductivity is concerned, the relationship between the declared value of thermal conductivity and the design value of thermal conductivity can be expressed as:

$$
\begin{aligned}
& \lambda_{2}=\lambda_{1} * F_{m} \\
& F_{m}=e^{\mathrm{f}_{\mathrm{U}}\left(\mathrm{U}_{2}-\mathrm{U}_{1}\right)}
\end{aligned}
$$

Where, $\lambda_{1}(\mathrm{~W} / \mathrm{m} . \mathrm{K})$ is the declared value of thermal conductivity, $\lambda_{2}(\mathrm{~W} / \mathrm{m} . \mathrm{K})$ is the design value of thermal conductivity, $f_{u}$ is the moisture conversion coefficient mass by mass, $\mathrm{u}_{1}$ is the moisture content mass by mass of the first set of conditions, $\mathrm{u}_{2}$ is the moisture content mass by mass of the second set of conditions.

\section{Material and Method}

\subsection{Materials}

The key physical and thermal properties of the Hemp and Stone Wool insulations tested are given in Table 1.

\begin{tabular}{|c|c|c|c|c|c|c|}
\hline Material & $\begin{array}{l}\text { Density } \\
\left(\mathrm{kg} / \mathrm{m}^{3}\right)\end{array}$ & $\begin{array}{l}\text { Thickness } \\
(\mathrm{mm})\end{array}$ & $\begin{array}{l}\text { Specific } \\
\text { heat } \\
\text { capacity } \\
(\mathrm{J} / \mathrm{kg} . \mathrm{K})\end{array}$ & Constituents & $\begin{array}{l}\text { Thermal } \\
\text { conductivity } \\
(\mathrm{W} / \mathrm{m} . \mathrm{K})\end{array}$ & $\begin{array}{l}\text { Vapour } \\
\text { permeability [26] } \\
(\mathrm{Kg} /(\mathrm{m} . \mathrm{S} . \mathrm{Pa}))\end{array}$ \\
\hline Hemp & 50 & 55 & 1600 & $\begin{array}{l}85 \% \text { Hemp fibres, } 10-12 \% \\
\text { bi-component fibres and } 3- \\
5 \% \text { soda }\end{array}$ & 0.038 & 0.56 \\
\hline
\end{tabular}

Table 1. Summary of the properties of the Hemp and Stone Wool insulations. 


$\begin{array}{lllllll}\begin{array}{l}\text { Stone } \\ \text { Wool }\end{array} & 23 & 100 & 850 & \begin{array}{l}\text { Amphibolite, about } 6 \% \\ \text { lime stone, about } 9 \% \\ \text { calcium oxide, resin }\end{array} & 0.038 & 0.46\end{array}$

Before installation, both Hemp and Stone Wool insulation materials were stored at 23 $( \pm 2)^{\circ} \mathrm{C}$ temperature and $50( \pm 5) \%$ relative humidity for 90 days. The adsorbed water contents in Hemp and Stone Wool for this exposure are $3.4( \pm 0.0) \mathrm{Kg} / \mathrm{m}^{3}$ and $0.1( \pm$ $0.001) \mathrm{Kg} / \mathrm{m}^{3}$, respectively, calculated from the adsorption-desorption isotherms determined in a DVS equipment (Fig. 1a). The desorption isotherms are not shown because of the very negligible hysteresis effect. Fig. 1b shows the water absorption curve of hemp in relation to time [4], the water absorption of Stone Wool is negligible and is not presented.
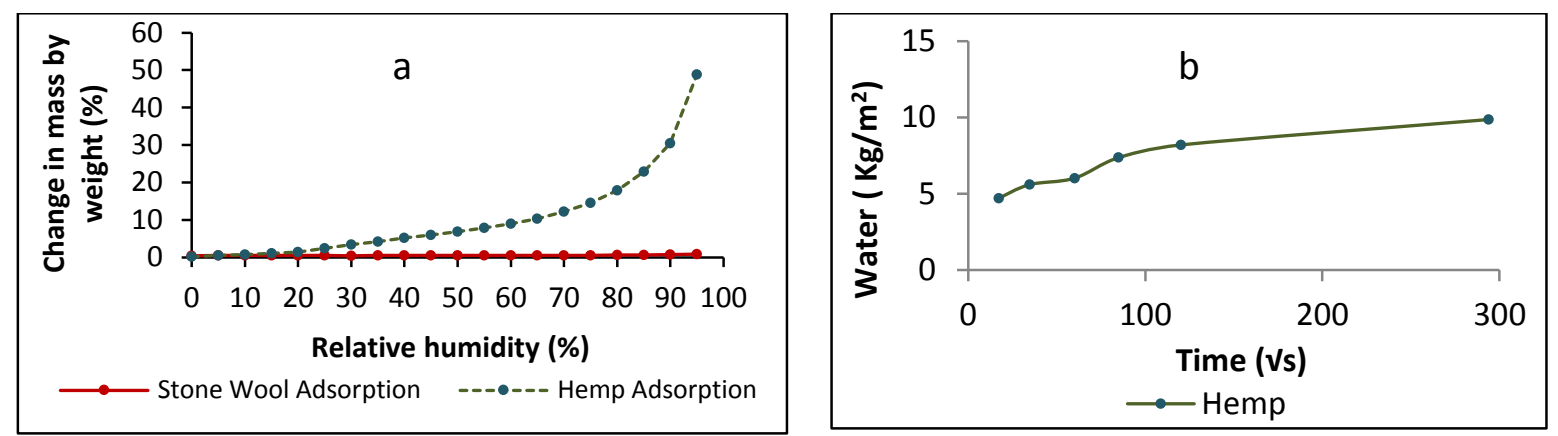

Fig. 1. (a) Adsorption isotherms of Hemp and Stone Wool insulation, (b) Water absorption of Hemp insulation [4].

The air permeability of the samples at 50 Pascal pressure difference was also measured using a DG-700 pressure and fan flow gauge, a cylindrical metal duct and a Duct Blaster fan. For the sample size of $400 \mathrm{~mm} \times 400 \mathrm{~m} \times 100 \mathrm{~m}$, the air permeability of Hemp and Stone Wool was $214 \mathrm{~m}^{3} / \mathrm{h}$ and $222 \mathrm{~m}^{3} / \mathrm{h}$, respectively.

\subsection{Instrumentation}

CS215 sensors were used to measure the temperature and relative humidity. The accuracy of the relative humidity measurement at $25^{\circ} \mathrm{C}$ is $\pm 4 \%$ over $0 \%-100 \%$ relative humidity. The length of the sensor is $180 \mathrm{~mm}$ and average diameter is $15 \mathrm{~mm}$. HFP01 
heat flux sensors were used to measure heat flux through the insulation. The measurement range is between $-2000 \mathrm{~W} / \mathrm{m}^{2}$ and $+2000 \mathrm{~W} / \mathrm{m}^{2}$ and the accuracy is \pm $5 \%$ on walls. The thickness of the sensor is $5 \mathrm{~mm}$ and the diameter is $80 \mathrm{~mm}$. A CR 1000 data logger was used to acquire sensor data.

\subsection{Method}

The tests were carried out using two different protocols of hygrothermal boundary conditions: quasi steady state and dynamic. For both protocols, the key objectives were to assess the moisture management potential and the equivalent thermal conductivity of the insulations using dual insulation set-ups. In the dual insulation setups, two insulations were placed adjacent to each other and were exposed to identical hygrothermal boundary conditions. To ensure unidirectional heat flux and moisture exposure, one surface of each insulation was exposed to a cold chamber and the other surface was exposed to a hot chamber with relative humidity control. The surface of the insulations facing the cold chamber was covered with a clear acrylic sheet to prevent any moisture interaction with the cold chamber and between the insulations. The acrylic outer surface also acted as an interface surface to induce and visualise condensation. The tests were carried out in two different laboratories in Europe following the quasi steady static and dynamic protocols. The design of the dual insulation setups and the hygrothermal conditioning devices varied between the protocols. The details of the individual protocols are provided in subsections 3.3 .1 and 3.3.2.

During both protocols, the vapour pressure inside the hot chamber was increased or decreased. Increase of vapour pressure pushed the moisture and enthalpy through the insulation towards the cold acrylic surface. The eventual decrease in vapour pressure caused the moisture to flow back to the hot chamber. It was assumed that 
the vapour pressure gradient would vary across the depth of the insulation materials, according to their vapour diffusion resistance factors and sorption isotherms. It was also assumed that condensation would occur earlier in the insulation-acrylic interface of the insulation that had lower hygric diffusivity, since hygric diffusivity is a function of moisture adsorption capacity and vapour permeability.

\subsubsection{Protocol for Test-1: Quasi Steady State}

In Test-1, the temperature difference between the opposite surfaces of the insulations was kept constant and step changes were made in the interior relative humidity of the hot chamber. Test-1 consisted of two tests: Test-1.1 and Test-1.2.

\section{Experimental Setup and Sample Installation}

Test 1.1: Hemp and Stone Wool insulations were placed inside an extruded polystyrene (XPS) insulation framework. The dimensions of the framework, the placement of insulations and the sensors are shown in Fig. 2. The XPS framework (Fig. 2a) was placed in an insulated steel partition frame. The steel partition frame was installed between the hot and cold chamber (Fig. 2b).

Temperature and relative humidity sensors were placed in the mid-thickness and on the cold side surface and on the warm side surface of the insulations. Heat flux sensors were placed on the acrylic sheets adjacent to the cold side surfaces of the insulation materials. 


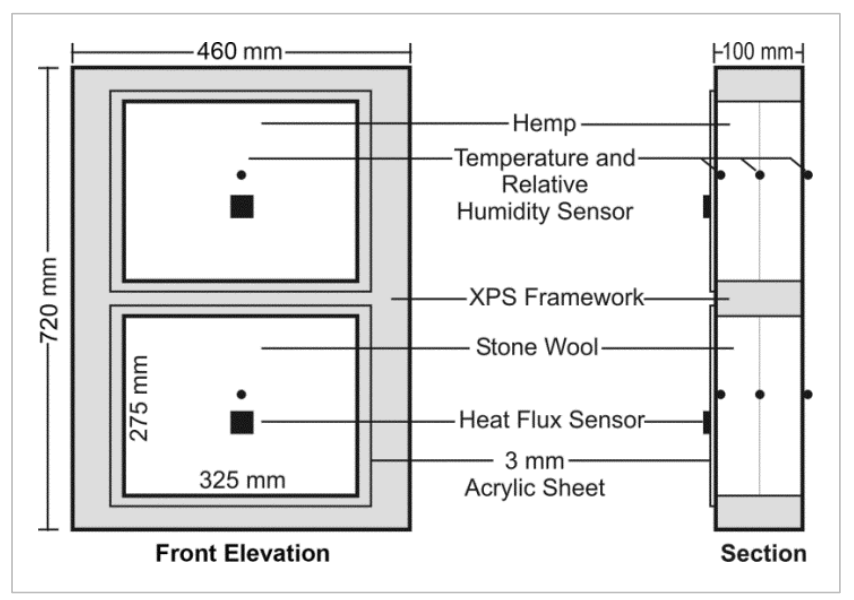

A

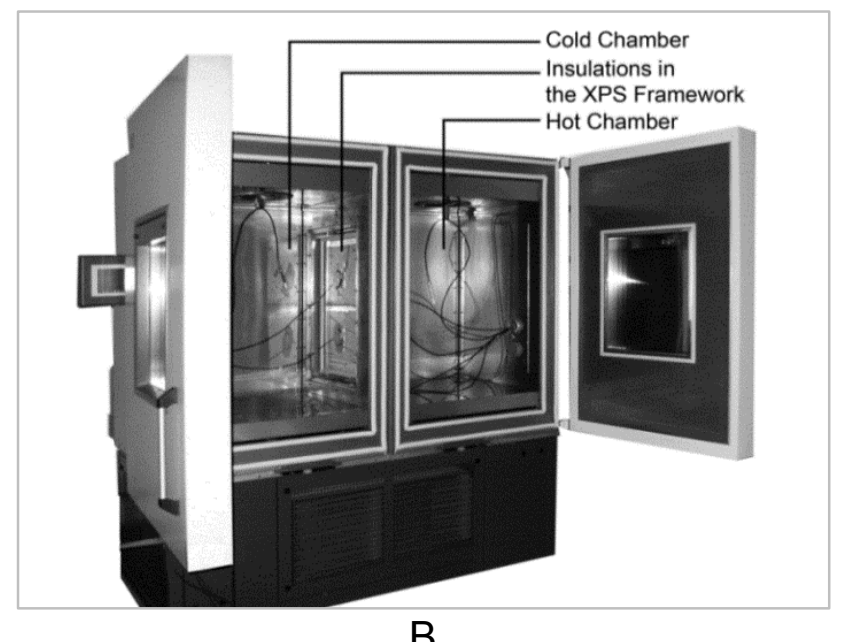

B

Fig. 2. (a) The front elevation and the cross section of the dual-insulation setup, (b) The dual climate chamber.

Test 1.2: In Test-1.2, heat flux sensors and relative humidity sensors were placed in the mid thickness of the insulations. Position of the insulations in the dual insulation setup was also swapped over vertically.

\section{Experimental method}

The target temperature and relative humidity profile for Test-1 is shown in Table 2 and Fig. 3. Temperature in the hot chamber was set at constant $23{ }^{\circ} \mathrm{C}$ and in the cold chamber at constant $7{ }^{\circ} \mathrm{C}$ resulting in a constant temperature difference of $16{ }^{\circ} \mathrm{C} \pm 1$ ${ }^{\circ} \mathrm{C}$. The following step changes in relative humidity were made in the hot chamber at 
every 24 hours: $33 \%, 55 \%, 80 \%, 95 \%, 55 \%$. However, another 8 hours were added to the initial step $(33 \%)$ during Test-1.1 so that the insulation materials were reasonably dry. These specific relative steps were selected for the following reasons: firstly, they are included in relative humidity variation protocols for standard adsorptiondesorption tests such as Nordtest [27] and ISO 24353 [28], secondly, the results can be compared with some of the in situ tests carried out using the same relative humidity variation protocol $[11,29]$. The time for each step of relative humidity condition was sufficient for the insulation materials to reach equilibrium moisture content. The relative humidity in the cold chamber, kept at $55 \%$, was not interacting with the cold surfaces of the insulations covered by acrylic sheets.

Table 2. The temperature and relative humidity profile of the climate chamber.

\begin{tabular}{llll|lll}
\hline \multicolumn{3}{c}{ Cold Chamber } & & & Hot Chamber & \\
\hline Steps & $\begin{array}{l}\text { Temperature } \\
\left({ }^{\circ} \mathrm{C}\right)\end{array}$ & $\begin{array}{l}\text { Relative } \\
\text { Humidity } \\
(\%)\end{array}$ & $\begin{array}{l}\text { Duration } \\
(\text { Hours })\end{array}$ & $\begin{array}{l}\text { Temperature } \\
\left({ }^{\circ} \mathrm{C}\right)\end{array}$ & $\begin{array}{l}\text { Relative } \\
\text { Humidity }(\%)\end{array}$ & $\begin{array}{l}\text { Duration } \\
(\text { Hours })\end{array}$ \\
\hline 1 & 7 & 55 & 33 & 23 & 33 & 33 \\
2 & 7 & 55 & 24 & 23 & 55 & 24 \\
3 & 7 & 55 & 24 & 23 & 80 & 24 \\
4 & 7 & 55 & 24 & 23 & 95 & 24 \\
5 & 7 & 55 & 24 & 23 & 55 & 24 \\
\hline
\end{tabular}

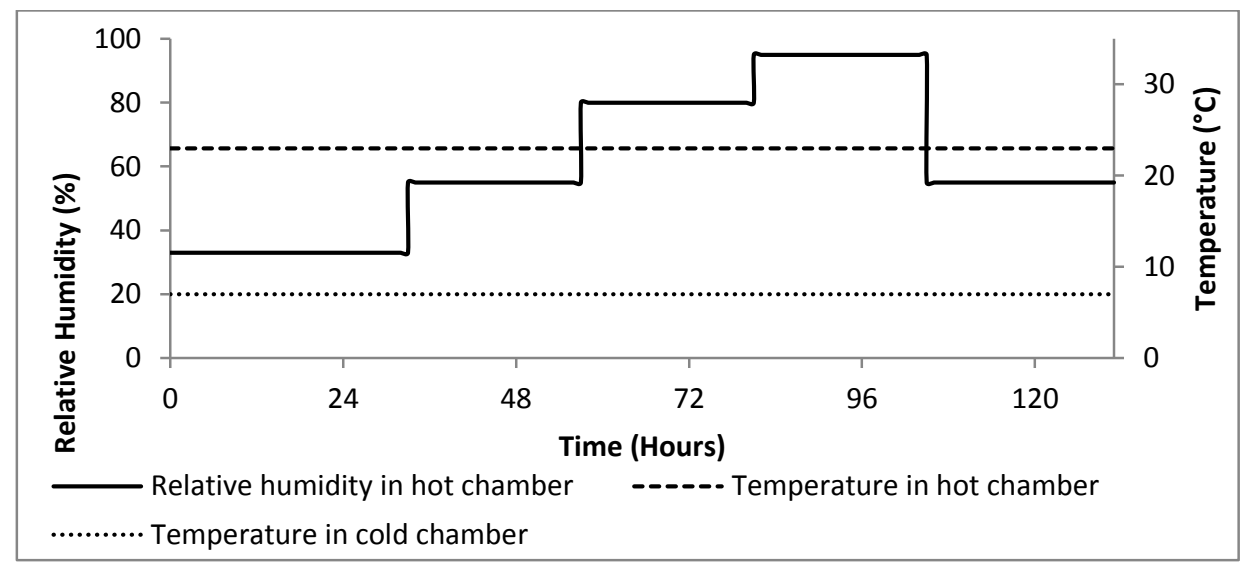

Fig. 3. The temperature and relative humidity profile of the climate chamber. 


\subsubsection{Protocol for Test-2: Dynamic State}

In Test-2, the temperature difference between the opposite surfaces of the insulations and the relative humidity of the hot chamber was dynamic.

\section{Experimental Setup and Sample Installation}

The insulations were placed side by side in a $30 \mathrm{~mm}$ EPS framework, separated by a $30 \mathrm{~mm}$ layer of EPS insulation (Fig. 4a). The EPS framework was placed inside an insulation holder in front of the hot chamber. The cold side surfaces of the insulations were covered by $3 \mathrm{~mm}$ clear acrylic sheets. Fig. $4 \mathrm{~b}$ shows the dynamic hygrothermal hot box setup.

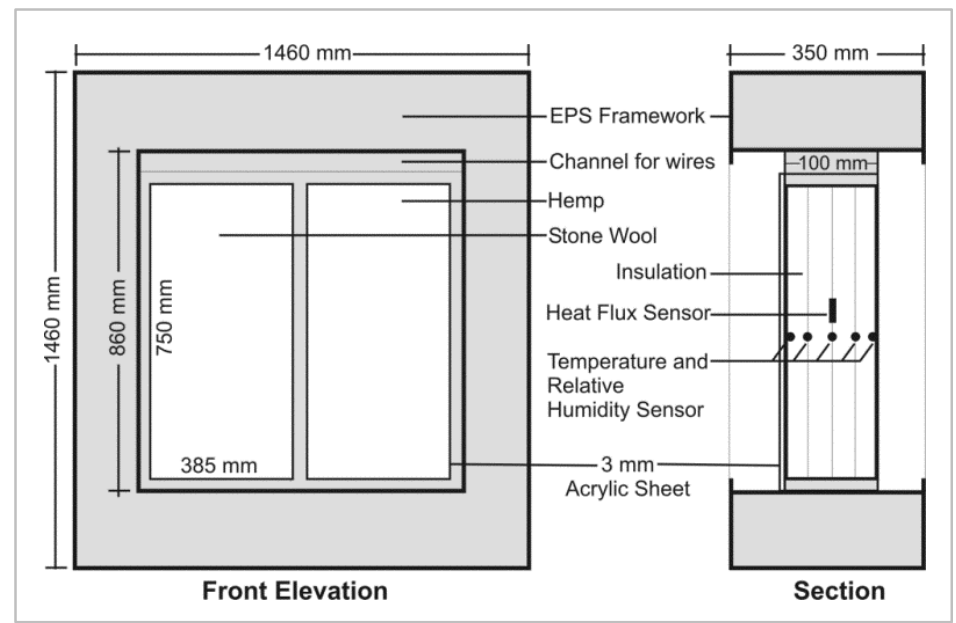

A

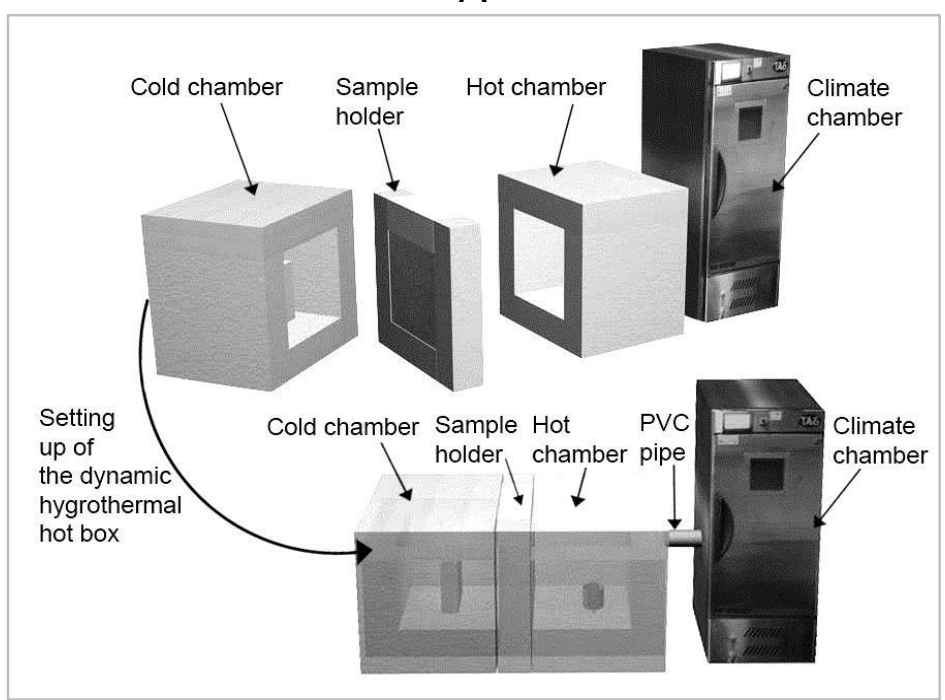


Fig. 4. (a) The front elevation and the section of the dual-insulation setup, (b) the hygrothermal hotbox setup.

Each insulation was fitted with one heat flux sensor and four temperature and relative humidity sensors. The position of the temperature and relative humidity sensors and heat flux sensors in the insulations is shown in Fig. 4a. The heat flux sensors were placed in the centre of the insulations. It was assumed that placing the heat flux sensor at the mid-thickness of the insulations would be most useful in acquiring the typical heat flux data, uninterrupted by the dynamic changes of temperature and relative humidity in the hot chamber. However, the heat flux sensors would be unable to register any sudden change in heat flux due to phase change in the insulation-acrylic interfaces. All data were logged in a CR 1000 data logger.

\section{Experimental method}

Hemp and Stone Wool insulation materials were exposed to the dynamic relative humidity ranges of the hot chamber varying between $35 \%$ and $80 \%$ as the dynamic hygrothermal hot box was not able to raise relative humidity above $80 \%$.To induce unidirectional heat flux within the temperature control range of the test setup, the internal temperature of the hot chamber was kept at $35{ }^{\circ} \mathrm{C}$ and the external temperature was kept at $19{ }^{\circ} \mathrm{C}$ with an amplitude of $3^{\circ} \mathrm{C}$. Two tests were conducted: Test-2.1 and Test-2.2. During Test-2.1, the relative humidity of the hygrothermal hot box was increased from $50 \%$ to $75 \%( \pm 5 \%)$ in 72 hours and then decreased from $75 \%$ to $50 \%( \pm 5 \%)$ in 24 hours. During Test-2.2, the relative humidity of the hot chamber was raised from $35 \%$ to $80 \%( \pm 5 \%)$ in 48 hours and then decreased from $80 \%$ to $35 \%$ $( \pm 5 \%)$ in 216 hours. The longer duration of Test-2.2 was made to study the drying out of the insulation materials. 


\section{Results and Discussion}

\subsection{Test 1: Quasi Steady State test}

\subsubsection{Moisture Management}

The relative humidity conditions in Hemp and Stone Wool insulations are shown in

Fig. 5. During Test-1.1, conducted in the winter time, air velocity adjacent to the exposed surfaces of Hemp and Stone Wool insulation was $0.45 \mathrm{~m} / \mathrm{s}$. However, during Test-1.2, conducted in the summer time, the air velocity near the exposed surface of Hemp was $1.2 \mathrm{~m} / \mathrm{s}$ and near the exposed surface of Stone Wool was $0.6 \mathrm{~m} / \mathrm{s}$. Since increase in air velocity decreases the surface air resistance and increases the rate of adsorption of moisture, it can be assumed that during Test-1.2 Hemp was subjected to higher moisture load.

During Test-1.1 (Fig. 5a), in response to the step changes of relative humidity in the hot chamber, the relative humidity in the internal surface, insulation-acrylic interface and the middle of the Stone Wool insulation changed instantaneously while the relative humidity in the middle and in the insulation-acrylic interface of Hemp insulation changed slowly. The relative humidity of Stone Wool-acrylic interface rose to $95 \%$ as soon as the relative humidity of the hot chamber was increased from $33 \%$ to $55 \%$. At the same time, the relative humidity of the Hemp-acrylic interface gradually increased to $72 \%$. When the relative humidity of the hot chamber was increased to $80 \%, 77$ hours after the beginning of the experiment, the relative humidity of Stone Wool-acrylic interface increased to $98.4 \%$ and the relative humidity of Hemp-acrylic interface increased to $84.3 \%$. When the relative humidity of the hot chamber was raised from $80 \%$ to $100 \%$, the relative humidity of Stone Wool-acrylic interface increased to $100 \%$, and the relative humidity at the Hemp-acrylic interface increased to $95 \%$. 

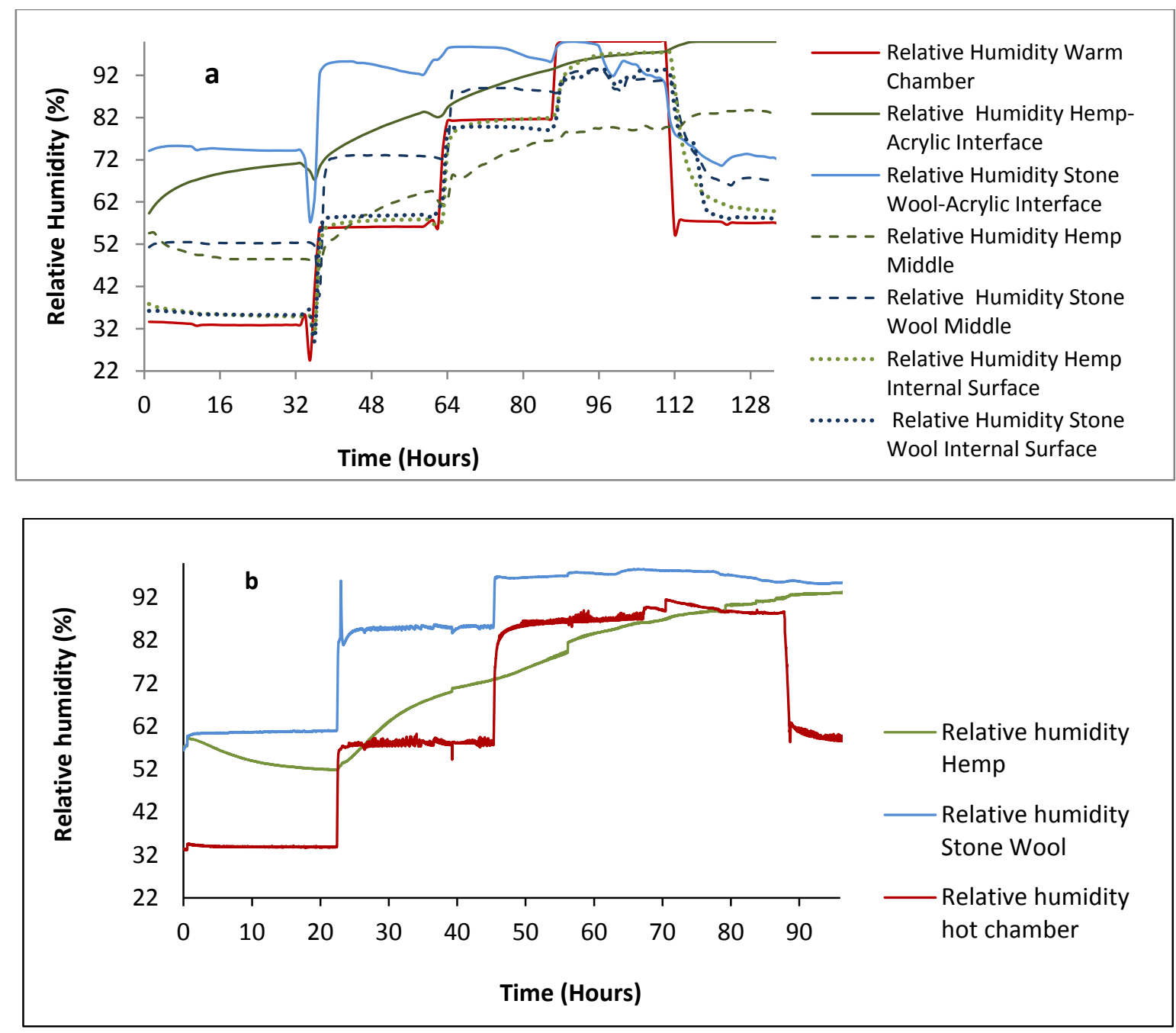

Fig. 5. Relative humidity (a) along the depth of Hemp and Stone Wool insulations during Test-1.1, (b) in the mid thickness of Hemp insulation during Test-1.2.

The relative humidity of Hemp-acrylic interface reached $100 \%$ only after the insulation was exposed to all the increasing step changes of relative humidity over 111 hours. While the middle of the Stone Wool insulation also responded instantly to the changes of relative humidity in the warm chamber, the middle of the Hemp insulation showed a dampened response to the relative humidity changes in the warm chamber. Similar phenomenon was also observed in the mid thickness of both insulations during Test1.2 (Fig. 5b). However, during Test-1.2, the relative humidity response of both insulations was at a higher magnitude due to the increased air velocity near the exposed surfaces of the insulations. 
Fig. 6 shows condensed water in the Stone Wool-acrylic interface and the dry Hempacrylic interface during Test-1.1. When the insulation materials were removed from the framework, water deposits on the lower surface of the framework of the Stone Wool insulation were also noticed, implying that further condensation had occurred in the Stone Wool insulation setup during the experiment. It was not possible to measure the amount of condensate resulting from the experiments but another study by the authors [29] did examine this aspect.

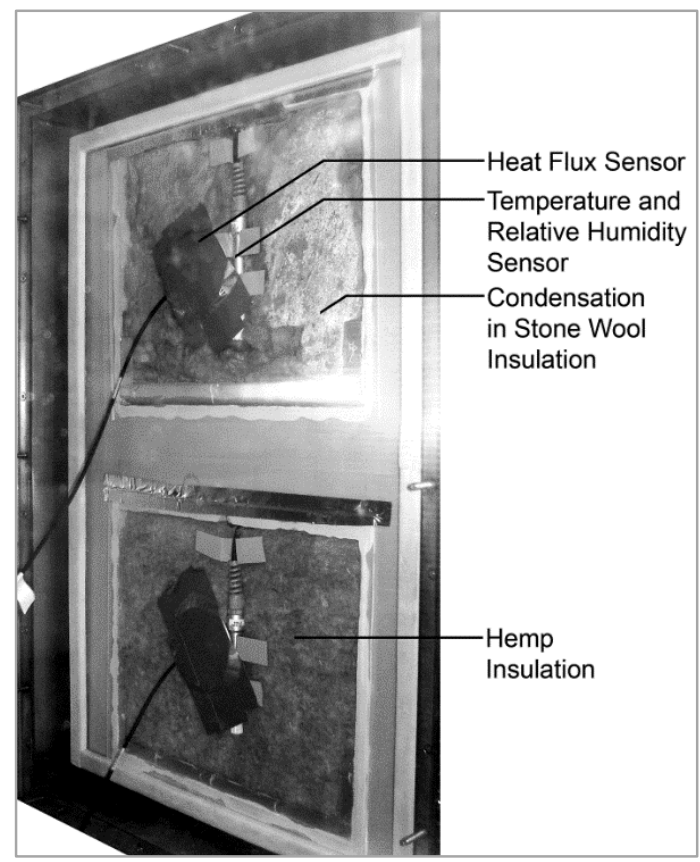

Fig. 6. Dew on acrylic inner surface of Stone Wool and dry acrylic inner surface of Hemp insulation.

Fig. 7 shows the internal surface temperature of the acrylic and the dew point temperature of insulation-acrylic interface air during Test-1.1. Condensation seemed to occur in the acrylic surface of the Stone Wool-acrylic interface as soon as the humidity of hot chamber increased from $33 \%$ to $55 \%$ and remained for $60( \pm 1)$ hours. Condensation seemed to occur in the acrylic surface of the Hemp-acrylic interface 36 $( \pm 1)$ hours later than it occurred in the Stone Wool-acrylic interface (Fig. 7). Hemp responded slowly to the decreasing step change in boundary relative humidity 
conditions. During Test 1.2, condensation also occurred earlier in the Stone Woolacrylic interface even though the air velocity near the exposed surface of Hemp was twice that near the exposed surface of Stone Wool.

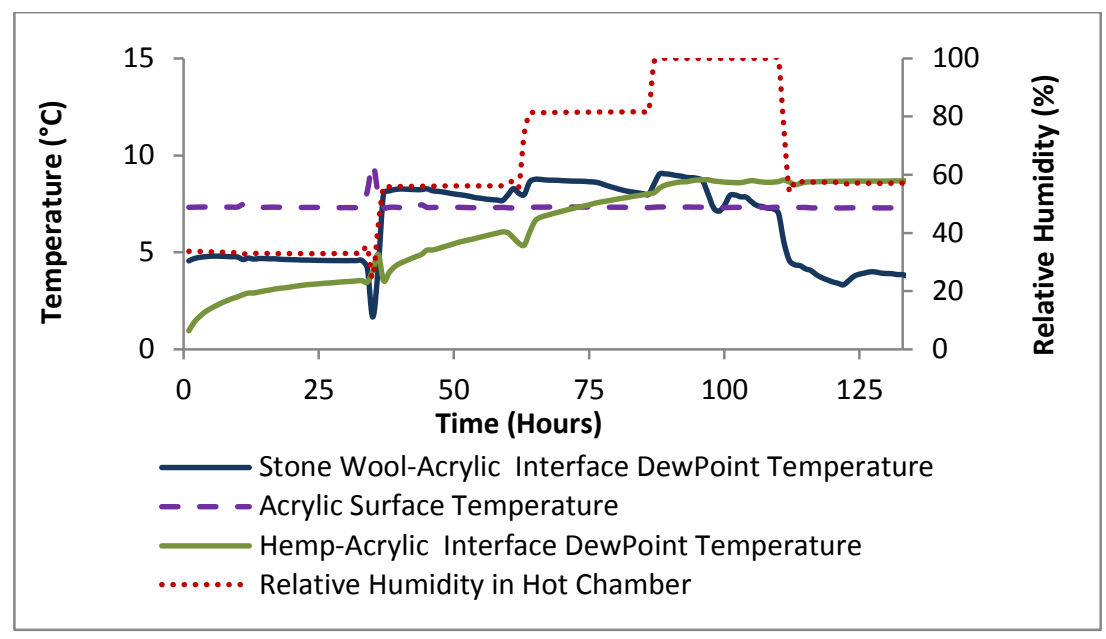

Fig. 7. Internal surface temperatures of the acrylic and the insulation-acrylic dew point temperatures.

During both tests, the temperature difference between the warm side and cold side of both insulations remained equal and constant most of the time. The temperature in the insulation-acrylic interfaces also remained nearly constant. Therefore, the rate of moisture flow at the Hemp-acrylic interface was lower than that at the Stone Woolacrylic interface during the experimental runs. Moisture flow is a function of hygroscopic capacity, vapour permeability and rate of air flow. Since the vapour permeability of both insulations is similar, as also observed by Collet et al. [30], and the cooler sides of the insulations were airtight, the managed response of Hemp insulation can mainly be attributed to its higher moisture adsorption capacity and to a limited extent to its relatively lower air permeability.

Once condensation occurred in the insulation-acrylic interface, the condensed water in touch with the insulation can be absorbed by the insulation. The amount of water that will be left on the surface of the acrylic will depend on the rate of condensation on 
the acrylic and the coefficient of water absorption and water absorption capacity of the insulation. Water absorption coefficient of Hemp insulation is $0.034 \mathrm{~kg} / \mathrm{m}^{2} \sqrt{\mathrm{s}}$ [26] and that of Stone Wool insulation is negligible. Therefore, Hemp will absorb more water than Stone Wool insulation when condensed water is in touch with the insulation surfaces.

\subsubsection{Heat flux and equivalent thermal conductivity}

The equivalent thermal conductivity values were determined from ambient temperature differences, heat flux and thickness of the material, following Equations 3 and 4 . The actual ranges of relative humidity for Tets-1.1 were: $33 \%, 56 \%, 81 \%$ and 100\% (Fig. 8a) and Test-1.2 were: $34 \%, 57 \%, 86 \%$ and $89 \%$ (Fig. 8b). It can be noticed in Fig. 8 that the temperature difference between the hot and cold chambers was constant throughout the duration of the experiment. Therefore, changes of equivalent thermal conductivity values of the insulation materials can be assumed to be due to the changes in relative humidity in the hot chamber. It can be observed in both Test 1.1 and Test 1.2 that the equivalent thermal conductivity of Hemp increases marginally with each increasing relative humidity range, which can be explained in terms of the equilibrium moisture content (EMC) of Hemp insulation. However, it can also be noticed that the equivalent thermal conductivity of Stone Wool insulation started decreasing from the onset of condensation at the $37^{\text {th }}$ hour during Test-1.1. Heat flux towards the cold chamber was expected to increase due to enthalpy flow and phase change. The reasons for this phenomenon can be the difference in the position of the heat flux sensor and the area of condensation on the acrylic surface or the distortion of heat flux measurement due condensation on the surface of the heat flux sensor. The heat flux sensor, due to its placement, plausibly failed to log the heat flux by enthalpy flow and phase change. There is also a possibility that once water condensed 
on the surface of the heat flux sensor, the oncoming heat was absorbed by the condensate and thus the heat flux sensor registered lower heat flux and the function of the sensor was distorted. Thus, the positioning of the heat flux sensor in the insulation-acrylic interface can cause uncertainty and distortion in the heat flux measurement when excessive condensation occurs in the interface. To address this issue, heat flux sensors were positioned in the mid thickness of the insulations during Test 1.2. During this test, both insulations exhibited an increase in thermal conductivity at high relative humidity ranges starting from $80 \%$ and onward (Fig.8b).
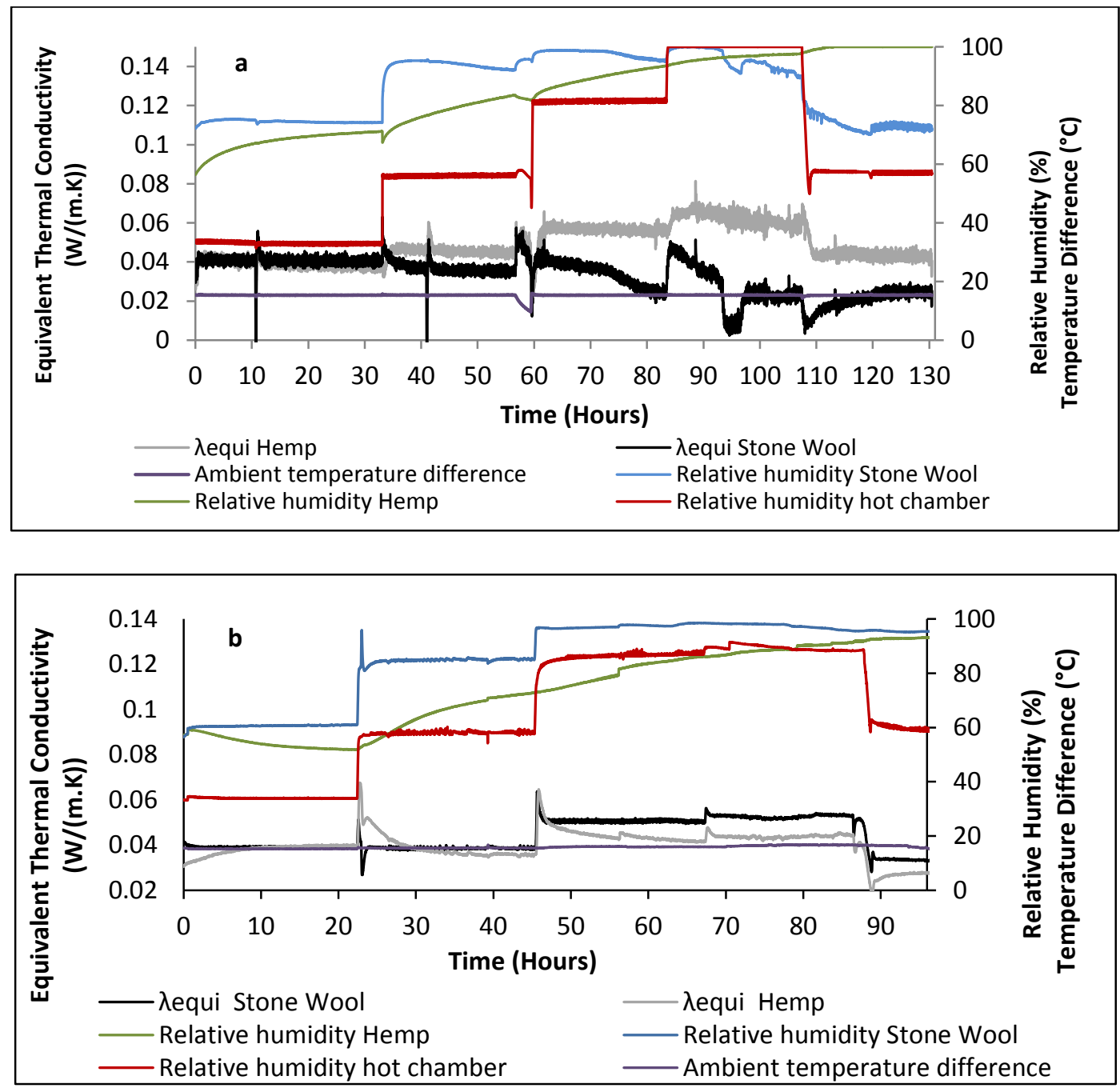


\section{Fig. 8. Equivalent thermal conductivity values of Hemp and Stone Wool insulation and interior and insulation-acrylic interface relative humidities during (a) Test-1.1 and (b) Test-1.2.}

Fig. 9 shows the thermal image of the insulations exposed to the final relative humidity step in the hot chamber during Test-1.2. In the Hemp insulation, a vertical gradient of moisture on the surface of the insulation is visible with gravity induced higher concentration towards the bottom. In the Stone Wool insulation, the hot and cold spots are randomly distributed, plausibly suggesting convective heat loss.

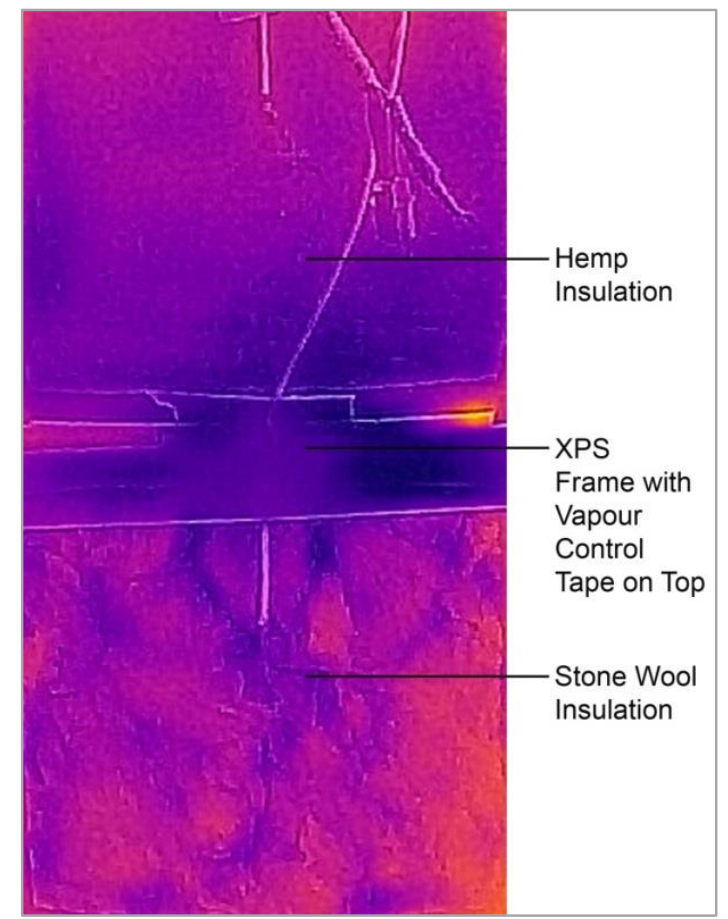

Fig. 9. Thermal image of the insulations facing the hot chamber.

Fig. 10 shows the thermal conductivity values of the insulations at different ranges of relative humidity and Table 3 and 4 compare the equivalent thermal conductivity with the corresponding design values of thermal conductivity determined by applying Equation 5. For determining design values of Hemp insulation, the moisture conversion coefficient has been taken as equal to that of the cellulose fibre. Moisture content by mass is determined from the adsorption isotherms of the insulations. The average of the whole data shows that the equivalent thermal conductivity of Hemp 
$(0.048 \mathrm{~W} /(\mathrm{m} . \mathrm{K}))$ is $16.6 \%$ higher than the design thermal value $(0.04 \mathrm{~W} /(\mathrm{m} . \mathrm{K}))$ at an average relative humidity of $63 \%$ during Test-1.1 and is equal to the design thermal value $(0.040 \mathrm{~W} /(\mathrm{m} . \mathrm{K}))$ at an average relative humidity of $66 \%$ during Test-1.2. The experimental average equivalent thermal conductivity value of Stone Wool $(0.033$ $\mathrm{W} /(\mathrm{m} . \mathrm{K}))$ is $17.5 \%$ lower than the design thermal value $(0.04 \mathrm{~W} / \mathrm{m}-\mathrm{K})$ during Test-1.1 and $9 \%$ higher than the design value during Test-1.2.
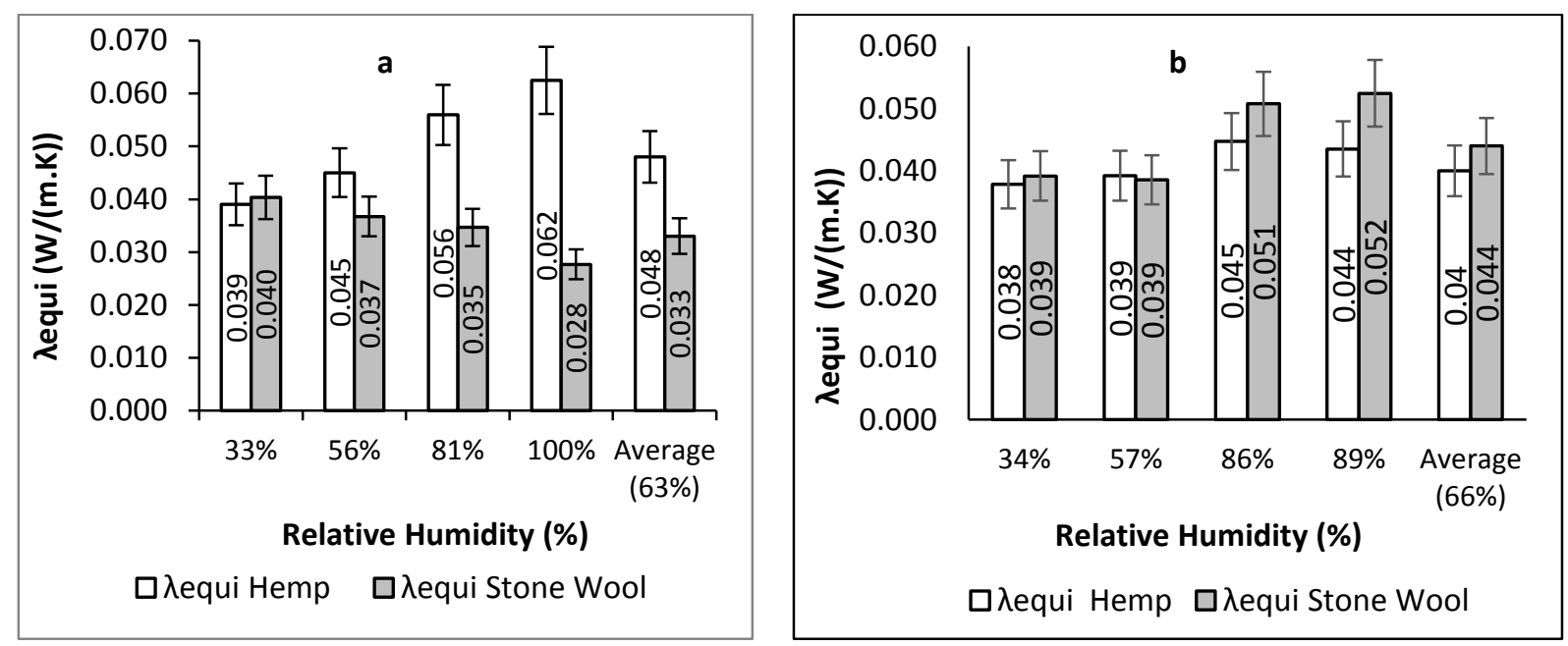

Fig. 10. Equivalent thermal conductivity values of Hemp and Stone Wool Insulations during (a) Test-1.1 and (b) Test-1.2.

Table 3. Experimental and design value of thermal conductivity with standard deviations (Test 1.1).

\begin{tabular}{|c|c|c|c|c|c|c|c|c|c|c|}
\hline $\begin{array}{l}\frac{\pi}{\pi} \\
\mathbb{0} \\
4 \\
0 \\
0 \\
\dot{2}\end{array}$ & 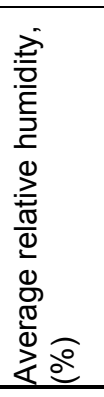 & 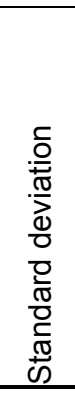 & 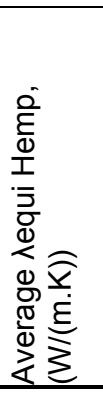 & 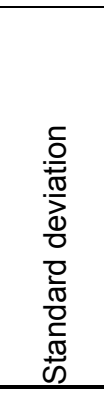 & 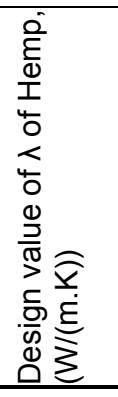 & 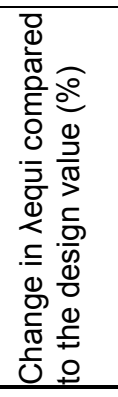 & 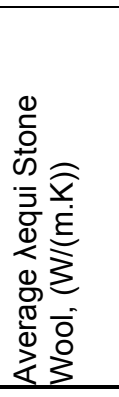 & 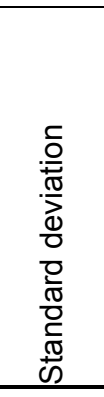 & 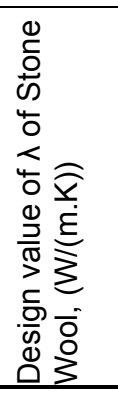 & 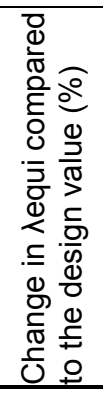 \\
\hline 1900 & 33.03 & 0.45 & 0.039 & 0.03 & 0.038 & 2.63 & 0.037 & 0.003 & 0.04 & \\
\hline 1300 & 56.06 & 0.41 & 0.045 & 0.004 & 0.038 & 18.42 & 0.034 & 0.004 & 0.04 & \\
\hline 1300 & 81.48 & 0.47 & 0.052 & 0.003 & 0.043 & 20.93 & 0.032 & 0.01 & 0.04 & \\
\hline 1300 & 100 & 0.03 & 0.062 & 0.003 & 0.05 & 24.00 & 0.026 & 0.01 & 0.04 & \\
\hline
\end{tabular}



deviations (Test 1.2).

\begin{tabular}{|c|c|c|c|c|c|c|c|c|c|c|}
\hline $\begin{array}{l}\frac{\pi}{0} \\
\frac{\pi}{0} \\
\text { to } \\
\dot{2} \\
\dot{z}\end{array}$ & 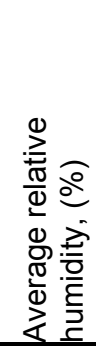 & 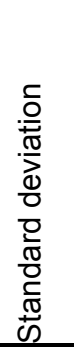 & 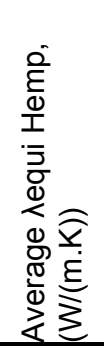 & 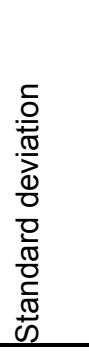 & 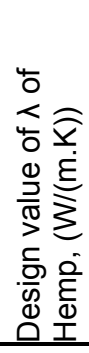 & 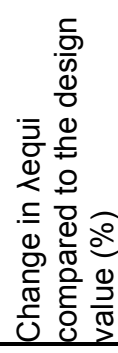 & 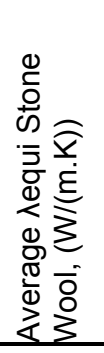 & 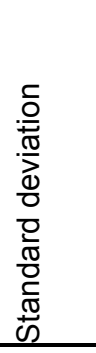 & 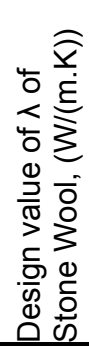 & 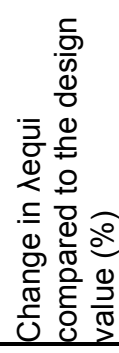 \\
\hline 1321 & 34 & 0.19 & 0.038 & 0.002 & 0.038 & 0 & 0.039 & 0.000 & 0.04 & -2.5 \\
\hline 1413 & 57 & 3.62 & 0.039 & 0.006 & 0.038 & 2.63 & 0.039 & 0.002 & 0.04 & -2.5 \\
\hline 1296 & 86 & 1.42 & 0.045 & 0.004 & 0.043 & 4.65 & 0.051 & 0.001 & 0.04 & 27.5 \\
\hline 1245 & 89 & 0.92 & 0.044 & 0.001 & 0.044 & 0 & 0.052 & 0.001 & 0.04 & 30 \\
\hline
\end{tabular}

\subsection{Test 2: Dynamic Tests}

\subsubsection{Moisture Management}

Fig.11 shows the relative humidity and vapour pressure in the insulation-acrylic interfaces and in the hot chamber during Test-2.1 and Test-2.2, respectively.
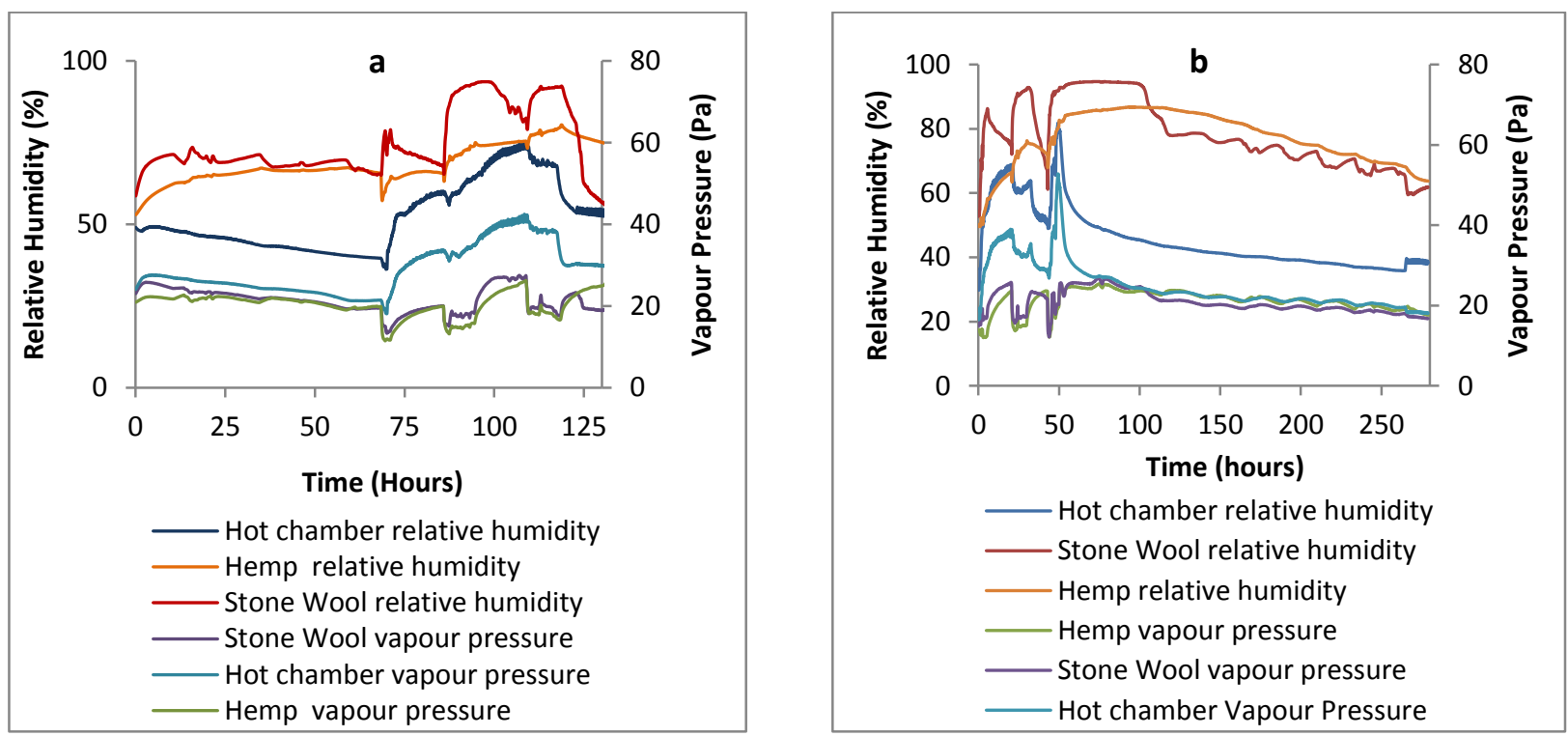

Fig. 11. Relative humidity and vapour pressure inside the dynamic hot box and in the insulation external surfaces during (a) Test-2.1 and (b) Test-2.2.

During Test-2.1, the relative humidity in the Stone Wool-acrylic interface increased to $90 \%$ in 30 hours in response to the rise in relative humidity in the hot chamber to $62 \%$. 
At the same time relative humidity in the Hemp-acrylic interface increased to $72 \%$. When the relative humidity in the hot chamber reduced, the relative humidity decreased quicker in the Stone Wool-acrylic interface than in the Hemp-acrylic interface. Similar observation about the insulation materials' response to changes in relative humidity can also be made about Test-2.2. During Test-2.2, heavy condensation in the Stone Wool-acrylic interface was noticed on the $46^{\text {th }}$ hour (Fig. 12). The relative humidity sensor registered about $93 \%$ relative humidity of the adjacent air.

The instances of occurrences of condensation at the acrylic surface can be estimated by determining its dew point temperature. Condensation is likely whenever the surface temperature of the acrylic is equal to or lower than the dew point temperature of the adjacent moist air.

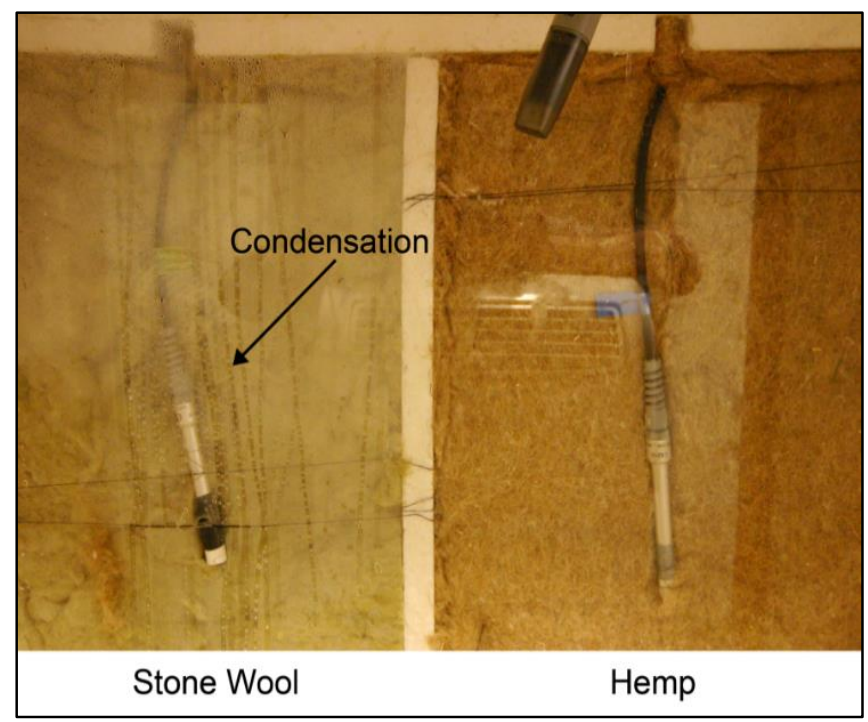

Fig. 12. Condensation in Stone Wool-acrylic interface, Hemp-acrylic interface remained dry.

It can be observed in Fig. 13 that condensation started on the Stone Wool-acrylic interface on the $57^{\text {th }}$ hour and carried on for 35 hours. This is marked by the grey shades between the line of acrylic surface temperature and the line of Stone Wool 
dew point temperature. The calculated period of condensation was confirmed by the visual observation of condensation on the acrylic surface of the Stone Wool-acrylic interface (Fig. 12).

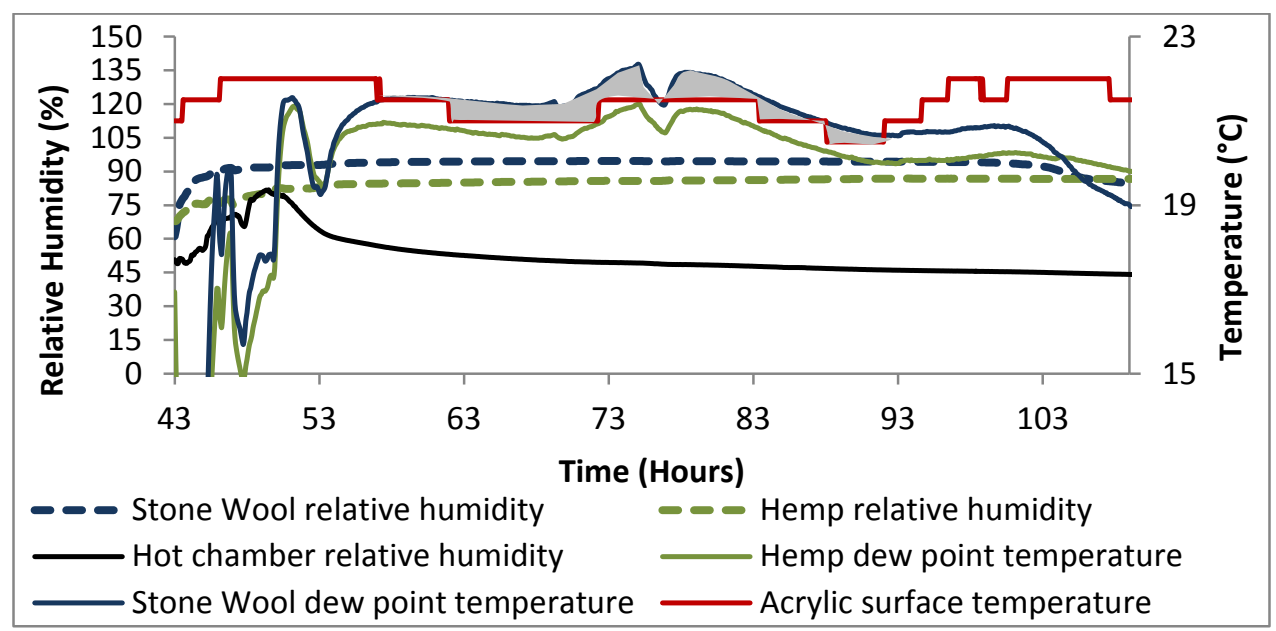

Fig. 13. Dew point temperatures of Hemp and Stone Wool and the acrylic surface temperature during condensation (Test-2.2).

Fig.14 shows the relative humidity at different depths of Stone Wool and Hemp insulation materials during Test-2.2. It can be noticed that peak relative humidity near the external surface of the Stone Wool insulation was $8 \%$ higher than the peak relative humidity near the external surface of Hemp insulation for 50 hours. When condensation was noticed during Test-2.2, the climate chamber was switched off on the $49^{\text {th }}$ hour so that the relative humidity inside the hot chamber could decrease. When the climate chamber was turned off, the relative humidity in the external surface of Stone Wool insulation remained $94.6 \%$ for 48 hours. The relative humidity in the external surface of Hemp insulation increased from $83 \%$ to $88 \%$ during the same period. However, at other depths, the relative humidity of Stone Wool insulation dropped instantaneously while Hemp showed dampened initial response. 


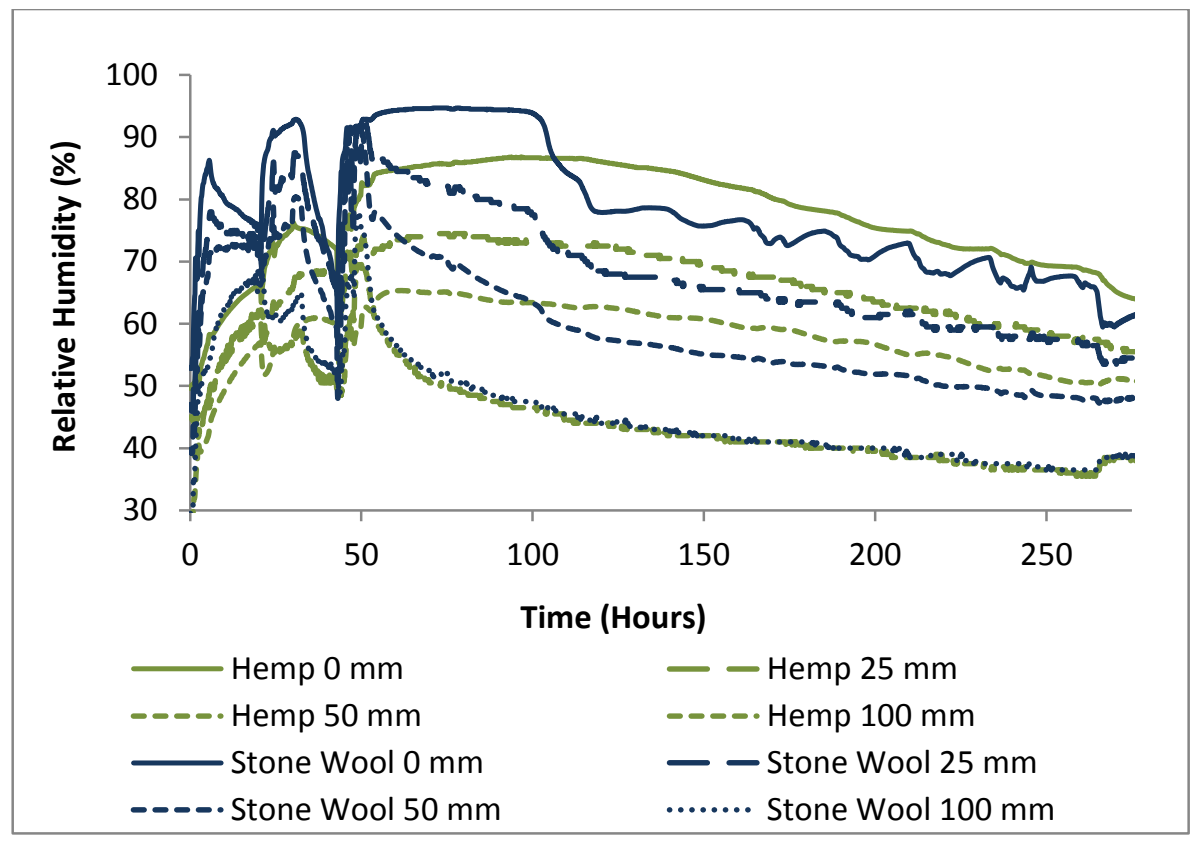

Fig. 14. Relative humidity distribution in the insulation materials during Test-2.

\subsubsection{Thermal Conductivity}

The effective equivalent thermal conductivity values of the insulations during Test-2.1 and Test-2.2 are shown in Fig. 15.
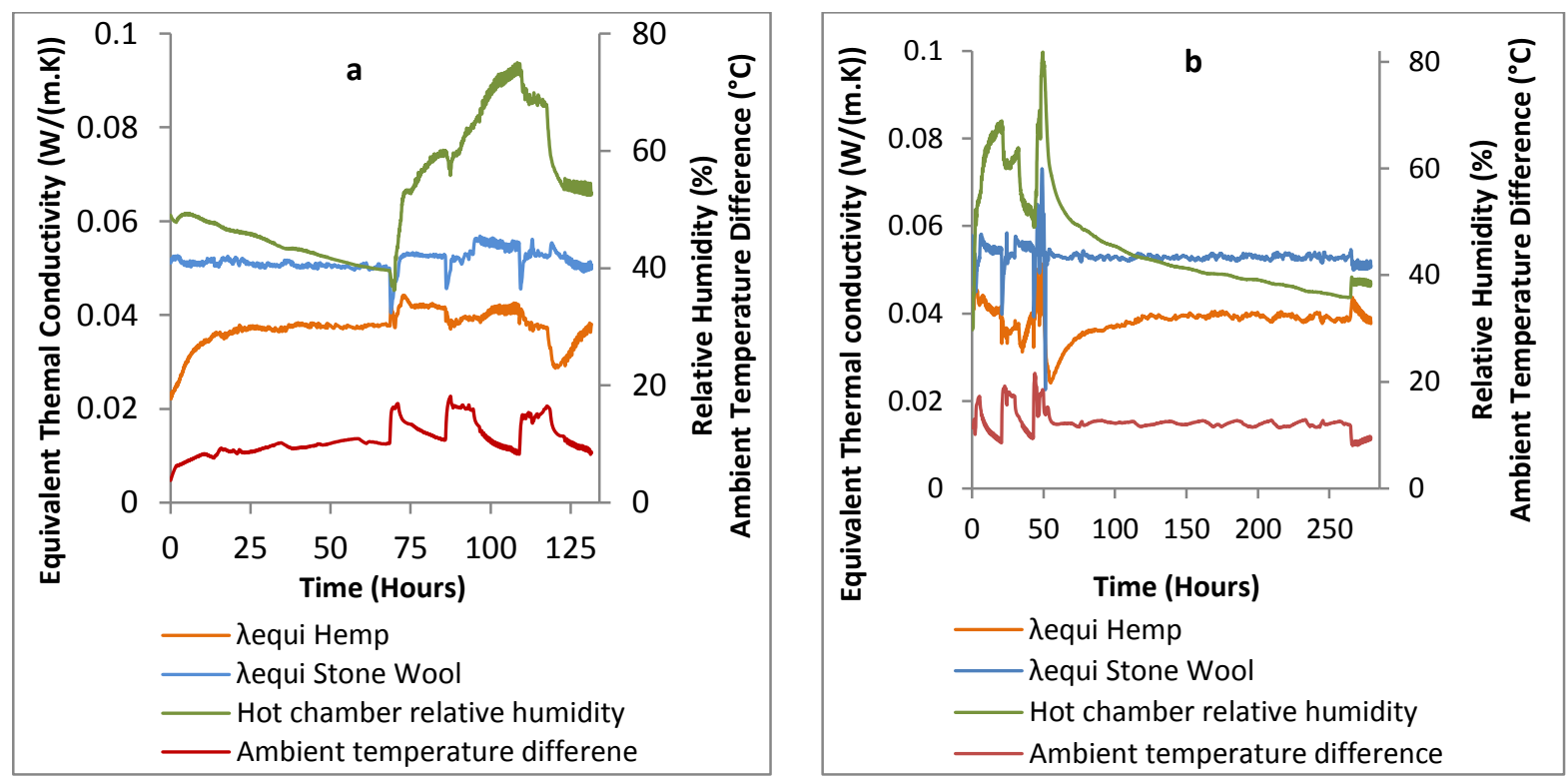

Fig. 15. Effective equivalent thermal conductivity of Hemp and Stone Wool insulations during (a) Test-2.1 and (b) Test-2.2.

The equivalent thermal conductivity values at ranges of relative humidity are presented in Fig. 16. It can be noticed that the average equivalent thermal conductivity values of 
Hemp insulation at $52 \%$ and $45 \%$ average relative humidity are similar to the manufacturers' declared thermal conductivity of Hemp insulation. While the equivalent thermal conductivity of Hemp at $80 \%$ relative humidity is $21 \%$ higher than the manufacturers' declared thermal conductivity (Fig. 16b), the increase was very transient. It suggests that if the Hemp insulation is exposed to fewer periods of high internal relative humidity compared to the periods of moderate internal relative humidity, then the effect of higher relative humidity on the average thermal conductivity of Hemp insulation is negligible. To ascertain moisture dependent thermal conductivity, adsorbed equilibrium moisture content for the ranges of relative humidity exposures needs to be determined. In an isothermal condition, a $100 \mathrm{~mm}$ thick fibrous insulation requires approximately 20 hours to reach equilibrium moisture content (EMC). In Test-2.1 and Test-2.2, due to the nature of the dynamic conditions, it was not possible to obtain continuous 20 hours data during the periods of peak relative humidity.
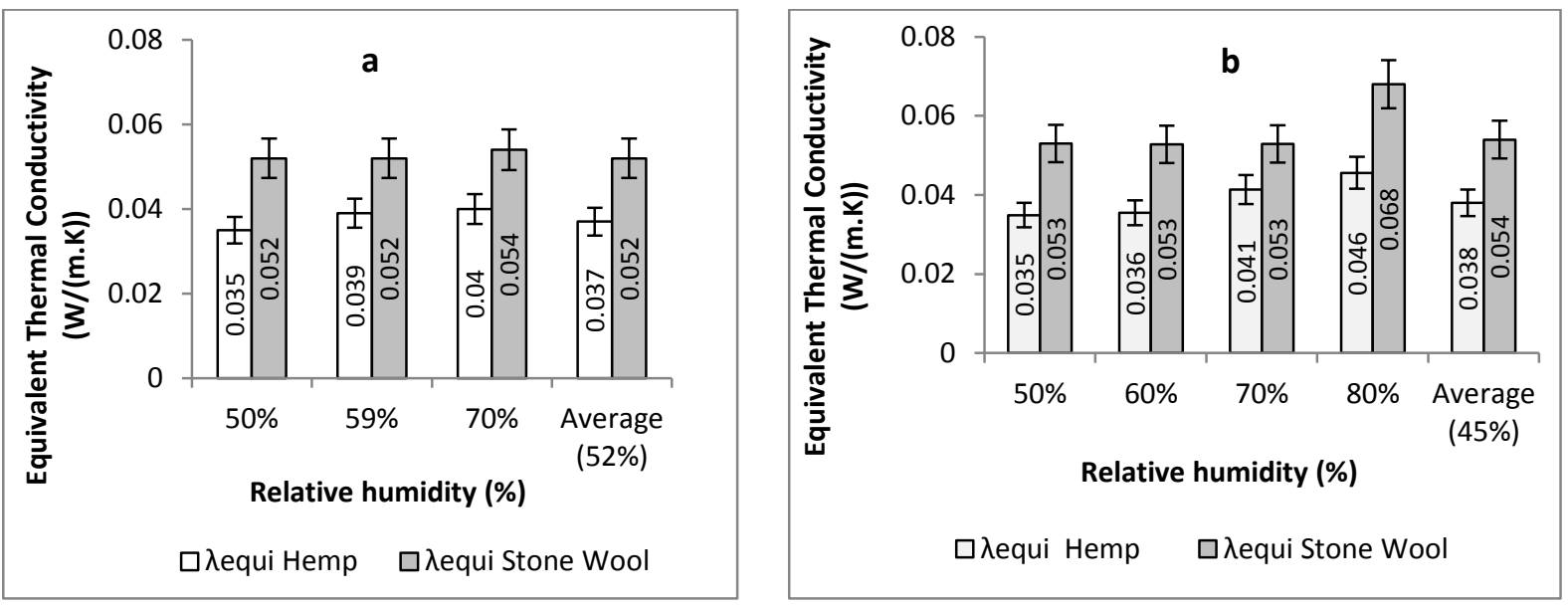

Fig. 16. Equivalent thermal conductivity values of Hemp and Stone Wool Insulations during (a) Test-2.1 and (b) Test-2.2.

For Stone Wool insulation, the average equivalent thermal conductivity is about 0.052 (Test-2.1) and 0.054 (Test-2.2) W/(m.K), about 42\% higher than the manufacturers' 
declared thermal conductivity. It is also explicit in Fig. 16(b) that thermal conductivity of Hemp insulation slightly increased with increased relative humidity while for Stone Wool insulation, thermal conductivity increased by $78.9 \%$ suddenly at $80 \%$ relative humidity. It implies that the heat loss through enthalpy flow and phase change may have occurred in Stone Wool insulation during Test-2.2 at $80 \%$ relative humidity, coinciding with the onset of condensation.

A table of equivalent thermal conductivity values along with the design values of the thermal conductivity of Hemp and Stone Wool insulation materials is presented in Table 5 and 6 . It can be observed that the experimental thermal conductivity value of Stone Wool $(0.054 \mathrm{~W} /(\mathrm{m} . \mathrm{K}))$ is higher than the design thermal value $(0.04 \mathrm{~W} /(\mathrm{m} . \mathrm{K}))$.

It can be further observed, in Table 5 and 6 , that the experimentally determined thermal conductivity values of Hemp insulation at $50 \%$ and $60 \%$ relative humidity are about $15 \%$ lower than the corresponding design values. For Stone Wool insulation, there is a substantial difference between experimental and design values of thermal conductivity. Since Stone Wool adsorbs negligible quantity of moisture, the value of the moisture supplement used to determine the design value of thermal conductivity is very low which results in low design values. However, in this experiment, the increase in heat flux through Stone Wool insulation may be due to the moisture transmission and phase change rather than adsorption. 
Table 5. Equivalent and design values of thermal conductivity of Hemp and Stone Wool insulations during Test-2.1.

\begin{tabular}{|c|c|c|c|c|c|c|c|c|c|}
\hline 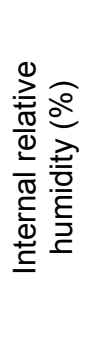 & $\begin{array}{l}\frac{\pi}{\pi} \\
\frac{\pi}{0} \\
4 \\
0 \\
0\end{array}$ & 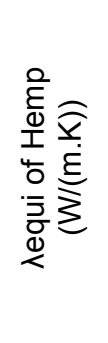 & 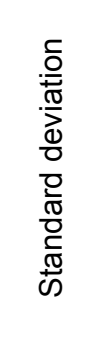 & 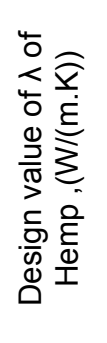 & 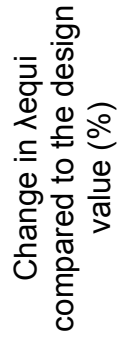 & 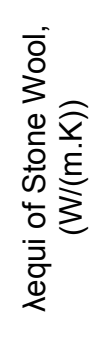 & $\begin{array}{l}\frac{0}{.0} \\
. \frac{0}{\pi} \\
\frac{0}{0} \\
\frac{0}{0} \\
\frac{0}{0} \\
\frac{0}{0} \\
\frac{0}{\pi} \\
\frac{\pi}{\omega}\end{array}$ & 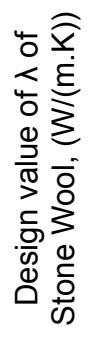 & 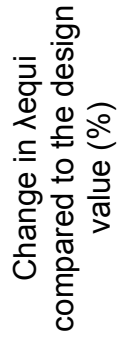 \\
\hline $50 \%$ & 465 & 0.035 & 0.005 & 0.04 & -12.5 & 0.052 & 0.001 & 0.04 & 30 \\
\hline $60 \%$ & 223 & 0.039 & 0.004 & 0.041 & -4.87 & 0.052 & 0.002 & 0.04 & 30 \\
\hline $70 \%$ & 243 & 0.04 & 0.002 & 0.041 & -2.43 & 0.054 & 0.002 & 0.04 & 35 \\
\hline
\end{tabular}

Table 6. Equivalent and design values of thermal conductivity of Hemp and Stone Wool insulations during Test-2.2.

\begin{tabular}{|c|c|c|c|c|c|c|c|c|c|}
\hline 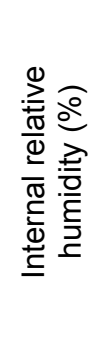 & $\begin{array}{l}\frac{\pi}{\pi} \\
\frac{\pi}{0} \\
40 \\
0 \\
0\end{array}$ & 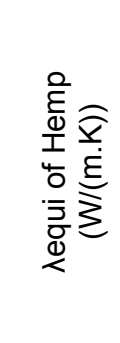 & 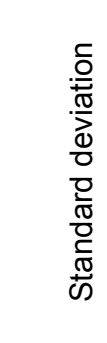 & 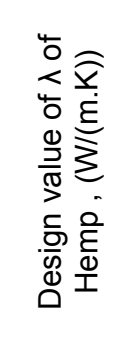 & 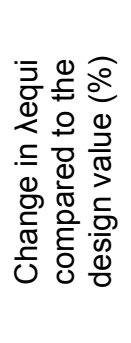 & 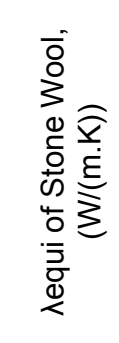 & 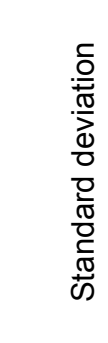 & 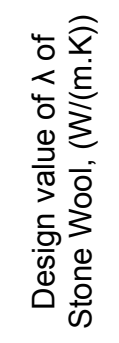 & 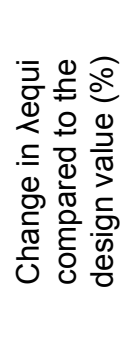 \\
\hline $50 \%$ & 392 & 0.035 & 0.006 & 0.04 & -12.5 & 0.053 & 0.005 & 0.04 & 32.5 \\
\hline $60 \%$ & 412 & 0.036 & 0.008 & 0.041 & -12.19 & 0.053 & 0.006 & 0.04 & 32.5 \\
\hline $70 \%$ & 80 & 0.041 & 0.011 & 0.041 & 0 & 0.053 & 0.012 & 0.04 & 32.5 \\
\hline $80 \%$ & 32 & 0.046 & 0.009 & 0.044 & 4.54 & 0.068 & 0.012 & 0.04 & 70 \\
\hline
\end{tabular}

\subsection{Summary and discussion of Test-1 and Test-2}

Moisture management: Both quasi steady state and dynamic tests show that Hemp insulation can dampen the moisture flux and reduce the risk of condensation while Stone Wool insulation is susceptible to moisture fluctuation and condensation. This observation corresponds with the in situ study of the moisture management of Hemp and Stone Wool insulations in a range of interior moisture load [11].The adsorption 
and desorption capacity of hemp fibre can potentially be used for moisture buffering of lofts [31] and interior space taking the effect of inner linings into account [32]. For Stone Wool insulation, high moisture content in the surface adjacent to the acrylic sheet, caused by condensation, and very high relative humidity in the mid depth also suggest that its adsorption isotherm does not represent the actual moisture content in the insulation during service conditions, as also observed by Vrána et al. [33].

Equivalent Thermal conductivity: Fig. 17 shows the relationship between the equivalent thermal conductivity values and the relative humidity in the hot chambers. Hemp insulation demonstrated moisture dependent increase in equivalent thermal conductivity both in quasi steady state and dynamic tests although values are often below design values. During the quasi steady state tests, the level of increase depended on the position of the heat flux sensor due to the influence of the phase change of moisture. When the heat flux sensor was placed in the mid thickness, the average equivalent thermal conductivity of Hemp insulation was $5 \%$ higher than the manufacturers' declared thermal conductivity. When the relative humidity and temperature were fluctuating during the dynamic tests, the average thermal conductivity of Hemp insulation was similar to the manufacturers' declared thermal conductivity.

For Stone Wool insulation, moisture flux dependent increase of thermal conductivity was observed during both quasi steady state and dynamic tests when the heat flux sensors were placed in the mid thickness of the insulations. The level of increase varied between the tests plausibly due to the variation in convective air flow near the exposed surface of the insulation. When the heat flux sensor was placed in the insulation-acrylic interface during Test-1.1 the thermal conductivity result was distorted. The distortion may had been due to the difference between the location of 
heat flux sensors and the location of condensation or due to the condensed water on the hat flux sensor absorbing the outgoing heat.
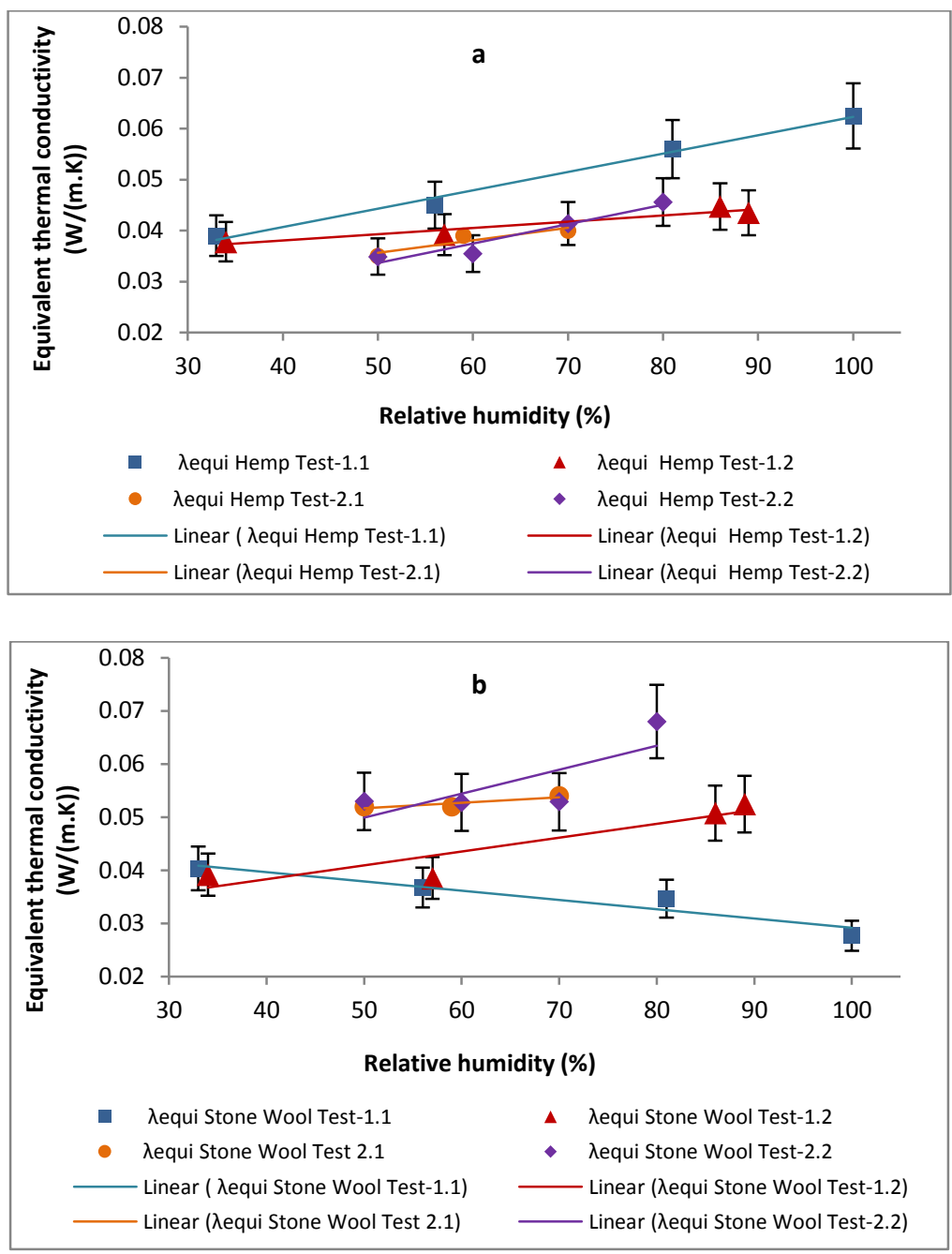

Fig. 17. Correlation between the relative humidity of hot chamber and the thermal conductivity of (a) Hemp and (b) Stone Wool insulations.

Although there are several factors contributing to the results including sensor position and occurrence of condensation, results suggest that thermal conductivity of hemp tends to be similar or lower than the manufacturers declared value under dynamic conditions, and higher only when high relative humidity levels are maintained over extended time periods. In the majority of heating climates external relative humidity and temperatures vary over diurnal time periods, and internal conditions vary according to the building construction and equipment and the actions of its occupants. 
Therefore it is not possible to state whether the protocols followed here do or do not correspond to conditions found in practice. However, given that climatic conditions tend to vary and overly humid interiors tend to get vented by the occupants, then probably the results derived under dynamic conditions are more relevant to practice.

\section{Conclusion}

Quasi steady state and dynamic hygrothermal experiments were carried out to assess the moisture and heat flux management capacity of Hemp and Stone Wool insulations. It was observed that, in response to the changes in relative humidity in the climate chamber, hygric response of Stone Wool insulation was instantaneous and that of Hemp insulation was delayed. It was further observed that compared to Stone Wool insulation, Hemp insulation could reduce the frequency and likelihood of condensation effectively. On the other hand, once vapour pressure was reduced, relative humidity decayed at a lower rate in Hemp insulation than in Stone Wool insulation. This property of hemp fibre may be utilised to maintain steady internal relative humidity by means of

moisture buffering. The better moisture management capacity of hemp insulation, combined with its carbon-negative credential, may potentially contribute to low-energy and robust vapour open construction system.

During the quasi steady state tests, the average equivalent thermal conductivity of Hemp insulation was $15.8 \%$ higher than the manufacturers' declared value and that of Stone Wool insulation was $1.3 \%$ higher than the manufacturers' declared value. During the dynamic tests, the average equivalent thermal conductivity of Hemp insulation was similar to manufacturers' declared value while that of Stone Wool insulation was $39.5 \%$ higher than manufacturers' declared value. Although the quasi state tests demonstrate the hygrothermal tolerance of the insulations, dynamic 
conditions are more representative of the reality. The hygrothermal behaviour of the insulations during the tests indicates that in a vapour open wall, the equivalent thermal conductivity in Stone Wool insulation is plausibly governed by enthalpy flow and phase change during condensation while the equivalent thermal conductivity in hemp insulation increases as a function of the increase in adsorbed moisture content.

\section{Acknowledgement}

The authors gratefully acknowledge the research grant provided by Innovate UK (formerly, the Technology Strategy Board, UK, DTI Project TP A0089J). The equipment support from the "Gheorghe Asachi" Technical University of lasi, Romania is also gratefully acknowledged.

\section{References}

[1] Labat M, Woloszyn M, Garnier G, Roux JJ. Dynamic coupling between vapour and heat transfer in wall assemblies: Analysis of measurements achieved under real climate. Building and Environment. 2015;87:129-41.

[2] Goto Y, Wakili KG, Ostermeyer Y, Frank T, Ando N, Wallbaum H. Preliminary investigation of a vapor-open envelope tailored for subtropical climate. Building and Environment. 2011;46(3):719-28.

[3] Pavlík Z, Černý R. Hygrothermal performance study of an innovative interior thermal insulation system. Applied Thermal Engineering. 2009;29(10):1941-6.

[4] Arnaud L. Comparative study of hygrothermal Performances of building materials. NOCMAT 2009: the 11th International Conference on Non-conventional Materials and Technologies. Bath, UK2009.

[5] Korjenic A, Petránek V, Zach J, Jitka H, J. Development and performance evaluation of natural thermal-insulation materials composed of renewable resources. Energy and Buildings. 2011;43:2518-23.

[6] Abdou A, Budaiwi I. The variation of thermal conductivity of fibrous insulation materials under different levels of moisture content. Construction and Building Materials. 2013;43:533-44.

[7] Clarke JA, Yaneske PP. A rational approach to the harmonisation of the thermal properties of building materials. Building and Environment. 2009;44(10):2046-55.

[8] Jerman M, Černý R. Effect of moisture content on heat and moisture transport and storage properties of thermal insulation materials. Energy and Buildings. 2012;53:3946.

[9] Vrána T, Gudmundsson K. Comparison of fibrous insulations - Cellulose and stone wool in terms of moisture properties resulting from condensation and ice formation. Construction and Building Materials. 2010;24(7):1151-7. 
[10] Vrána T, Björk F. A laboratory equipment for the study of moisture processes in thermal insulation materials when placed in a temperature field. Construction and Building Materials. 2008;22(12):2335-44.

[11] Latif E, Ciupala MA, Wijeyesekerac DC. The comparative in situ hygrothermal performance of Hemp and Stone Wool insulations in vapour open timber frame wall panels. Construction and Building Materials. 2014;73:205-13.

[12] Nicolajsen A. Thermal transmittance of a cellulose loose-fill insulation material. Building and Environment. 2005;40:pp. 907-14.

[13] Southern JR. Summer condensation within dry lined solid walls. Building Services Engineering Research and Technology. 1986;7(3):101-6.

[14] Derome D, Saneinejad S. Inward vapor diffusion due to high temperature gradients in experimentally tested large-scale wall assemblies. Building and Environment. 2010;45(12):2790-7.

[15] Rasmussen TV, Nicolajsen A. Assessment of the performance of organic and mineral-based insulation products used in exterior walls and attics in dwellings. Building and Environment. 2007;42:829-39.

[16] Toman J, Vimmrová A, Černý R. Long-term on-site assessment of hygrothermal performance of interior thermal insulation system without water vapour barrier. Energy and Buildings. 2009;41(1):51-5.

[17] Walker R, Pavía S. Thermal performance of a selection of insulation materials suitable for historic buildings. Building and Environment. 2015;94:155-65.

[18] Shea A, Lawrence M, Walker P. Hygrothermal performance of an experimental hemp-lime building. Construction and Building Materials. 2012;36:270-5.

[19] McClung R, Ge H, Straube J, Wang J. Hygrothermal performance of crosslaminated timber wall assemblies with built-in moisture: field measurements and simulations. Building and Environment. 2014;71:95-110.

[20] Stazi F, Tittarelli F, Politi G, Di Perna C, Munafò P. Assessment of the actual hygrothermal performance of glass mineral wool insulation applied 25 years ago in masonry cavity walls. Energy and Buildings. 2014;68:292-304.

[21] IUPAC. Compendium of Chemical Terminology2012.

[22] Institute BS. BS EN ISO 12572. Hygrothermal performance of building materials and products -Determination of water vapour transmission properties. London: BSI; 2000.

[23] ISO 9869. Thermal insulation-Building elements -In-situ measurement of thermal resistance and thermal transmittance. Switzerland: International Organization for Standardization; 1994.

[24] Domínguez-Muñoz F, Anderson B, Cejudo-López JM, Carrillo-Andrés A. Uncertainty in the thermal conductivity of insulation materials. Energy and Buildings. 2010;42(11):2159-68.

[25] ISO 10051. Thermal Insulation-Moisture effect on heat transfer- Determination of thermal transmissivity of a moist material. Switzerland: International Organization for Standardization; 1995.

[26] Latif E, Tucker S, Ciupala MA, Wiyjeyesekera DC, Newport D. Hygric properties of hemp bio-insulations with differing compositions. Construction and Building Materials. 2014;66(C):702-11.

[27] Rode C. Moisture buffering of building materials. Technical University of Denmark; 2005.

[28] ISO 24353. Hygrothermal performance of building materials and products -Determination of moisture adsorption/desorption properties in response to humidity variation. Switzerland: International Organization for Standardization; 2008. 
[29] Latif E, Ciupala MA, Tucker S, Wijeyesekera DC, Newport DJ. Hygrothermal performance of wood-hemp insulation in timber frame wall panels with and without a vapour barrier. Building and Environment. 2015;92:122-34.

[30] Collet F, Achchaq F, Djellab K, Marmoret BH. Water vapor properties of two hemp wools manufactured with different treatments. Construction and Building Materials. 2011;25:1079-85.

[31] Tucker S, Latif E, C. Wijeyesekera D. An experimental study of moisture buffering of bio-insulations in lofts. Structural Survey. 2014;32(5):434-48.

[32] Latif E, Lawrence M, Shea A, Walker P. Moisture buffer potential of experimental wall assemblies incorporating formulated hemp-lime. Building and Environment. 2015;93:199-209.

[33] Vrána T, Björk F. Frost formation and condensation in stone-wool insulations. Construction and Building Materials. 2009;23(5):1775-87. 